\title{
Full-field Digital Image Correlation with Kriging Regression
}

\author{
Dezhi Wang ${ }^{1}$, F. A. DiazDelaO ${ }^{1,2}$, Weizhuo Wang ${ }^{3}$ \\ and John E Mottershead ${ }^{1,2}$ \\ ${ }^{1}$ Centre for Engineering Dynamics, School of Engineering, University of Liverpool, \\ L69 3GH, United Kingdom. \\ ${ }^{2}$ Institute for Risk and Uncertainty, University of Liverpool, L69 3GH, \\ United Kingdom. \\ ${ }^{3}$ School of Engineering, Manchester Metropolitan University, \\ Manchester M1 5GD, United Kingdom
}

\begin{abstract}
A full-field Digital Image Correlation (DIC) method with integrated Kriging regression is presented in this paper. The displacement field is formulated as a best linear unbiased model that includes the correlations between all the locations in the Region of Interest (RoI). A global error factor is employed to extend conventional Kriging interpolation to quantify displacement errors of the control points. An updating strategy for the self-adaptive control grid is developed on basis of the Mean Squared Error (MSE) determined from the Kriging model. Kriging DIC is shown to outperform several other full-field DIC methods when using open-access experimental data. Numerical examples are used to demonstrate the robustness of Kriging DIC to different choices of initial control points and to speckle pattern variability. Finally Kriging DIC is tested on an experimental example.
\end{abstract}

Keywords: Full-field, Digital Image Correlation, Kriging Regression.

\section{Introduction}

Over the past three decades several different methods have been developed and successfully applied in Digital Image Correlation (DIC). These methods belong to two general classes, i.e. local (subset-based) methods and global (full-field) techniques, both of which have been used extensively in different applications. The local approach is perhaps the better established of the two because of its simplicity and suitability to parallel computation [1], but lacks inter-subset continuity and is more sensitive to measurement noise than the global approach. Consequently it is necessary to apply smoothing as a post-processing operation to measured displacements before computing strain results [2]. Alternatively, the global approach imposes certain constraints and treats the Region of Interest (RoI) as a whole, thereby enabling 
smooth displacement fields to be achieved together with good sub-pixel accuracy. The same level of sub-pixel accuracy is achievable by the global approach, more efficiently than the local approach, which requires subset overlapping [3] with multiple processing of the same data and increased computational cost.

Full-field DIC methods include: Finite Element (FE) based methods [4-9]; the Extended FE method, known as XFEM, [10-13]; B-Spline methods (NURBS) [14, 15] and Spectral methods based on spatial Fourier transforms [16-18]. DIC techniques aim to produce an accurate and reliable displacement field through the computed correlation of deformed speckle patterns with a reference image. This process requires the use of shape functions to describe the displacement field in terms of grey-scale values determined in terms of individual pixel intensities within a subset or RoI. Of course, it is generally not possible to design a shape function that perfectly matches the actual displacement field in a particular application. However, the Kriging prediction has the advantage that is based not only upon regressing certain parameters on discrete measurements, but also on the correlation of neighbouring samples. The fitting residual is represented by a Gaussian random process resulting in a best linear unbiased prediction. This represents lack of knowledge of the true displacement field and is not related to measurement error. The choice of a Gaussian random process is analogous to the choice of a Gaussian random variable in statistics: it is analytically tractable, flexible and frequently correct. Unlike other full-field shape functions that normally require an artificial control grid, the Kriging formula can generate the control grid for a RoI automatically on the basis of its estimated Mean Square Error (MSE). In addition it is possible to adapt the Kriging formula to account for imperfect sample-point data due to measurement noise that would otherwise be reproduced exactly (by conventional Kriging interpolation) because of perfect correlation of the sample point with itself. This adaptation, known as Kriging regression, will be described in detail in Section 3.

In this paper Kriging regression is integrated into the classical full-field DIC algorithm. The full-field displacement estimate is obtained by training the Kriging model using increasing numbers of sample (or control) points at each step until the MSE at untried sites (between the control points) is deemed to be acceptably small. At the end of this process the displacements at the untried sites are found in terms of the complete system of control-point displacements. Kriging regression generally outperforms the classical FE and B-spline methods where untried-site displacements are determined only in terms of several neighbouring control points. Figure 1(a) illustrates this point, where it is seen that the inner-point displacement is determined by only 4 nodal displacements when using the Q4-FE shape function [19], possibly resulting in abrupt ridges at the element boundaries. When using the B-spline method, the inner-point displacement, shown in Figure 1(b), is given in terms of a greater number of nodal displacements, but shape-function remains local to the inner point. The Kriging shape function is genuinely global, as shown in Figure 1(c) where the inner-point displacement is given in terms control points distributed over the entire RoI.

In this study, three case studies are used to test the performance of the proposed Kriging DIC method. In the first example, a two-directional rigid body translation is applied and a fixed regular control grid is used. This example provides the basis for a comparison of Kriging DIC with Q4-FE DIC and Cubic Spline DIC. In the second 
case study using irregular, adaptive control grids and FE-generated displacement fields, based on different numerical generation of speckle patterns, the robustness of Kriging DIC to initially chosen control points and speckle pattern variability is tested. Finally, in the third case study Kriging DIC is applied to a full-scale experimental structure and results are compared to those obtained from a commercial DIC system.

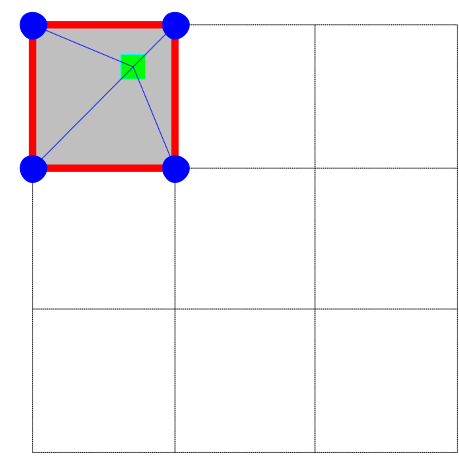

(a)

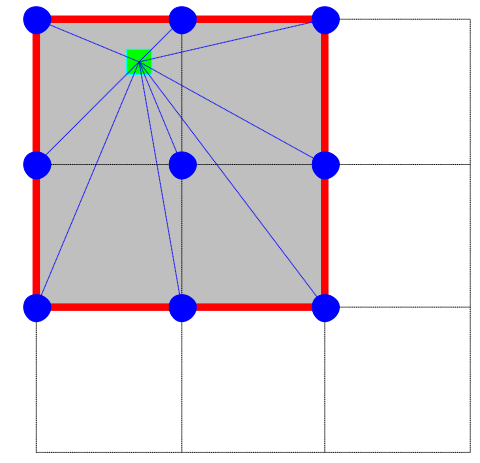

(b)

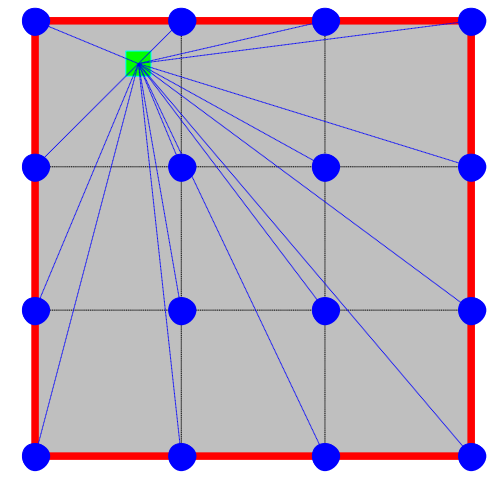

(c)

Figure 1 Dependency relationship of one inner point (green square): (a) Q4-FE, (b) Cubic Spline, (c) Kriging - control points shown as blue circles.

\section{Review of the Full-field DIC Method}

Full-field DIC is considered for the case of a two-dimensional image where the unknown displacement field $(u(x, y), v(x, y))$ is to be determined at spatial coordinate $(x, y)$. The displacement $(u(x, y), v(x, y))$ may also be understood as the optical flow from a reference image $f(x, y)$ of speckle-pattern intensity to its corresponding deformed image $g(x, y)$. Then the displacement field may be estimated by minimising the objective function,

$$
\eta(u, v)=\int_{\Theta}(g(x+u(x, y), y+v(x, y))-f(x, y))^{2} \mathrm{~d} \Theta
$$

where $\Theta$ denotes the region of interest (RoI) in the reference image.

In practice, the continuous displacement field $(u(x, y), v(x, y))$ may be approximated by a linear combination of basis functions of finite dimension $n$, expressed as

$$
\begin{aligned}
& u(x, y) \approx \sum_{j=1}^{n} \mu_{j}(x, y) \rho_{u_{j}} \\
& v(x, y) \approx \sum_{j=1}^{n} \mu_{j}(x, y) \rho_{v_{j}}
\end{aligned}
$$


where $\mu_{j}(x, y) ; j=1,2, \ldots, n$ are the kernel functions and $\rho_{u_{j}}, \rho_{v_{j}} ; j=1,2, \ldots, n$ are the combination coefficients. Since $g(x+u(x, y), y+v(x, y))$ is an implicit function of $(u(x, y), v(x, y))$, the Newton method may be applied to solve the minimisation problem. Therefore, an approximate solution of the full-field displacement, $(u(x, y), v(x, y))$, may be obtained by iteration $[5,6,15,20]$

$$
\mathbf{M}_{w}^{i}\left(\boldsymbol{\rho}_{w}^{i+1}-\boldsymbol{\rho}_{w}^{i}\right)=\mathbf{b}_{w}^{i} ; \quad w \in\{u, v\}
$$

where $\mathbf{M}_{u}^{i}, \mathbf{M}_{v}^{i}$ are $n \times n$ matrices and $\mathbf{b}_{u}^{i}, \mathbf{b}_{v}^{i}$ are $n \times 1$ vectors, with components given by

$$
\begin{aligned}
\left(m_{j k}\right)_{w}^{i}= & \int_{\Theta}\left(\mu_{j}(x, y) \frac{\partial g\left(x+u^{i}, y+v^{i}\right)}{\partial z}\right) \\
& \times\left(\mu_{k}(x, y) \frac{\partial g\left(x+u^{i}, y+v^{i}\right)}{\partial z}\right) \mathrm{d} \Theta
\end{aligned}
$$

and

$$
\begin{aligned}
\left(b_{j}\right)_{w}^{i}= & \int_{\Theta} \mu_{j}(x, y) \frac{\partial g\left(x+u^{i}, y+v^{i}\right)}{\partial z} \\
& \times\left(f(x, y)-g\left(x+u^{i}, y+v^{i}\right)\right) \mathrm{d} \Theta
\end{aligned}
$$

where $z \in\{x, y\}$ when $w \in\{u, v\}$ respectively and $j, k=1,2, \ldots, n$.

The gradients $\frac{\partial g\left(x+u^{i}, y+v^{i}\right)}{\partial z}$ and $\frac{\partial g\left(x+u^{i}, y+v^{i}\right)}{\partial z}$ in equations (4) and (5) are in principle updated at each iteration. However, as proposed by Sutton [21, 22], the grey-level gradients may be calculated from the reference image rather than the deformed image without loss of accuracy i.e. $\frac{\partial g\left(x+u^{i}, y+v^{i}\right)}{\partial z}=\frac{\partial f(x, y)}{\partial z}$.

The interpolation functions in Equation (2) are generally local piecewise functions [14, 23]. e.g. cubic spline or finite element shape functions. The combination coefficients then represent the displacements of a set of control points (or nodes). In this paper, a different linear modelling approach for the displacement field is investigated, known as Kriging regression.

\section{Kriging-DIC}

The obtained displacement from Equation (2) and (3) is an approximate solution on a linear subspace. In this Section, the approximation residual 
$e_{w}(x, y)=w(x, y)-\sum_{j=1}^{n} \mu_{j}(x, y) \rho_{w_{j}}, w \in\{u, v\}$, in the DIC algorithm will be modelled as a Gaussian random field.

The true displacement field $w(x, y)$ may be modelled as a realisation of a random function $W(x, y)$ which combines a deterministic regression model and a zero-mean stochastic field as [24],

$$
W(x, y)=\sum_{\ell=1}^{m} c_{\ell}(x, y) \beta_{\ell}+Z(x, y)
$$

where $c_{\ell}(x, y), \ell=1, \ldots, m$, are the regression functions and $\beta_{\ell}$ denotes the $\ell^{\text {th }}$ regression parameter and $Z(x, y)$ is a Gaussian stochastic field with zero mean and covariance $\operatorname{cov}\left(Z\left(x_{j}, y_{j}\right), Z\left(x_{k}, y_{k}\right)\right)$ between control points $j$ and $k$, which is assumed to take the form

$$
\operatorname{cov}\left(Z_{j}, Z_{k}\right)=\sigma_{w}^{2} r_{j k}\left(\mathbf{x}_{j}, \mathbf{x}_{k}, \vartheta_{x}, \vartheta_{y}\right) ; \quad \mathbf{x}_{j}=\left(\begin{array}{ll}
x_{j} & y_{j}
\end{array}\right)^{T} ; \quad \mathbf{x}_{k}=\left(\begin{array}{ll}
x_{k} & y_{k}
\end{array}\right)^{T}
$$

where $r_{j k}\left(\mathbf{x}_{j}, \mathbf{x}_{k}, \vartheta\right)=\operatorname{corr}\left(Z_{j}, Z_{k}\right)$ is determined by the proximity of points $j$ and $k$ and correlation parameters $\vartheta_{x}, \vartheta_{y}$, and $Z\left(x_{j}, y_{j}\right), Z\left(x_{k}, y_{k}\right)$ are abbreviated to $Z_{j}, Z_{k}$. The term $\sigma_{w}^{2}$ is a field variance, one value for each component of the displacement response, $w \in\{u, v\}$.

Denoting $\mathbf{\rho}=\left[w_{1}, \cdots, w_{n}\right]^{\mathrm{T}}$ as the displacements of a set of control points $\left(x_{j}, y_{j}\right), j=1,2, \ldots, n$, , the Kriging method may be used to obtain a Best Linear Unbiased Prediction (BLUP) for the displacement field [25], expressed as

$$
\hat{w}(x, y)=\sum_{j=1}^{n} \kappa_{j}(x, y) \rho_{j}=\boldsymbol{\kappa}^{T} \boldsymbol{\rho}
$$

where $\kappa_{j}(x, y), j=1,2, \ldots, n$, are the Kriging weights [23] obtained by the unbiasedness and minimisation of mean squared error (MSE),

$$
\begin{aligned}
& \underset{\kappa}{\arg \min } .\left\{\mathrm{E}\left[(w(x, y)-\hat{w}(x, y))^{2}\right]\right\} \\
& \text { subject to } \mathrm{E}[w(x, y)-\hat{w}(x, y)]=0
\end{aligned}
$$

where $E[\bullet]$ denotes the mathematical expectation. The Kriging weights may then be written as [25], 


$$
\boldsymbol{\kappa}(x, y)=\mathbf{R}^{-1}\left(\mathbf{r}(x, y)-\mathbf{C}\left(\mathbf{C}^{T} \mathbf{R}^{-1} \mathbf{C}\right)^{-1}\left(\mathbf{C}^{T} \mathbf{R}^{-1} \mathbf{r}(x, y)-\mathbf{c}(x, y)\right)\right)
$$

where $\boldsymbol{\kappa}(x, y)=\left(\begin{array}{llll}\kappa_{1} & \kappa_{2} & \cdots & \kappa_{n}\end{array}\right)^{T}, \mathbf{R}$ is the matrix of control-point displacement correlation functions, $r_{j k} ; \mathbf{r}(x, y)$ is the correlation vector of displacement between a dependent location $(x, y)$ (a non-control point) and all the control points $\left(x_{j}, y_{j}\right), j=1,2, \ldots, n ; \mathbf{C}$ is a 'design' matrix consisting of regression function values evaluated at control points, $c_{j \ell}=\mathbf{c}_{\ell}\left(x_{j}, y_{j}\right)$; and $\mathbf{c}$ is the vector of regression functions, $c_{\ell}=c_{\ell}(x, y)$.

It can be easily verified that the sample-point displacements are exactly reproduced by the Kriging model (8), (10). Let $w(x, y)=w_{j}$, then $\mathbf{r}(x, y)=\mathbf{R}_{:, j}$ so that $\mathbf{R}^{-1} \mathbf{r} x, y=\mathbf{e}_{j}$. Also $\mathbf{c}(x, y)=\mathbf{C}^{T} \mathbf{e}_{j}$ which causes the term $\left(\mathbf{C}^{T} \mathbf{R}^{-1} \mathbf{r}(x, y)-\mathbf{c}(x, y)\right)$ to vanish. Then it is seen that $\boldsymbol{\kappa}(x, y)=\mathbf{e}_{j}$.

However, in DIC the sample data are not measured with perfect accuracy, but are subject to measurement noise and imprecision [26-29], the effect of which might be reduced by pre-filtering [30, 31]. However, in this study it can be accounted for in a global sense by perturbing the correlation matrix which is replaced in Equation (10) (and in subsequent equations) by $\mathbf{R}+e_{M}^{2} \mathbf{I}$ where $e_{M}^{2}$ predominantly represents measurement error (but also error induced by other sources such as numerical error), considered to be independent and identically distributed at each sample point, hence the identity matrix in the added term $e_{M}^{2} \mathbf{I}$. This modified formulation is known as Kriging regression [29] (as opposed to Kriging interpolation, which is the conventional formulation that predicts the sample points exactly) and introduces an additional parameter $e_{M}^{2}$ to be determined.

The parameters of the Kriging regression model $\left\{\boldsymbol{\beta}, \sigma_{w}^{2}, \vartheta_{x}, \vartheta_{y}, e_{M}^{2}\right\}$, $\boldsymbol{\beta}=\left(\begin{array}{llll}\beta_{1} & \beta_{2} & \cdots & \beta_{m}\end{array}\right)$ may be solved by maximizing the log likelihood of the observed data $\boldsymbol{\rho}$. For an assumed Gaussian distribution [32, 33], and ignoring constant terms, this may be expressed as,

$$
-\frac{n}{2} \ln \left(\sigma_{w}^{2}\right)-\frac{1}{2} \ln \left(\left|\mathbf{R}\left(\vartheta_{x}, \vartheta_{y}, e_{M}^{2}\right)\right|\right)-\frac{(\boldsymbol{\rho}-\mathbf{C} \boldsymbol{\beta})^{\mathrm{T}} \mathbf{R}\left(\vartheta_{x}, \vartheta_{y} e_{M}^{2}\right)^{-1}(\boldsymbol{\rho}-\mathbf{C} \boldsymbol{\beta})}{2 \sigma_{w}^{2}}
$$

In practice the concentrated log likelihood function [32] is used, given by,

$$
\frac{n}{2} \ln \left(\hat{\sigma}_{w}^{2}\right)+\frac{1}{2} \ln \left(\left|\mathbf{R}\left(\vartheta_{x}, \vartheta_{y}, e_{M}^{2}\right)\right|\right)
$$


where $(\hat{\bullet})$ denotes an estimate. The log likelihood function (12) is complex and generally multimodal. Thus, the computation of optimal values for $\vartheta_{x}, \vartheta_{y}$ and $e_{M}^{2}$ usually requires specialised optimisation algorithms and heuristics like genetic algorithms [24, 34] or gradient-free methods such as the Hooke and Jeeve's algorithm [35], and the Nelder-Mead [36] simplex algorithm. The latter was employed in this work.

The estimate $\hat{\sigma}_{w}^{2}$ is given by,

$$
\hat{\sigma}_{w}^{2}=\frac{1}{n}(\boldsymbol{\rho}-\mathbf{C} \hat{\boldsymbol{\beta}})^{T} \mathbf{R}^{-1}\left(\vartheta_{x}, \vartheta_{y}, e_{M}^{2}\right)(\boldsymbol{\rho}-\mathbf{C} \hat{\boldsymbol{\beta}})
$$

with $\hat{\boldsymbol{\beta}}$ the least-squares solution of the weighted system of equations,

$$
\mathbf{R}^{-1 / 2} \mathbf{C} \boldsymbol{\beta}=\mathbf{R}^{-1 / 2} \boldsymbol{\rho}
$$

The introduction of $e_{M}^{2}$ has the benefit of acting as a regularisation parameter [29] against ill-conditioning of the correlation matrix, which tends to prevail when large numbers of control points are introduced.

In this paper a first-order regression function is chosen. Thus referring to Equation (6),

$$
\begin{gathered}
c_{1}(x, y)=1, c_{2}(x, y)=x_{1}, \cdots, c_{n+1}(x, y)=x_{n}, \\
c_{n+2}(x, y)=y_{1}, \cdots, c_{2 n+1}(x, y)=y_{n}
\end{gathered}
$$

so that $m=2 n+1$. Consequently Equation (14) is under-determined, so that a minimum-norm solution is obtained for $\hat{\boldsymbol{\beta}}$.

Also the correlation function is assumed exponential (also called Gaussian), expressed in the form,

$$
r_{j k}=\exp \left(-\vartheta_{x}\left(x_{j}-x_{k}\right)^{2}-\vartheta_{y}\left(y_{j}-y_{k}\right)^{2}\right)
$$

The choice of this correlation function relies on the assumption of the response surface inferred by the Kriging regression to be smooth. This means that points close to each other have a higher correlation. The terms $\vartheta_{x}$ and $\vartheta_{y}$ determine how far apart both $x_{j}$ and $x_{k}$ and $y_{j}$ and $y_{k}$ need to be before differences in the estimate given by equation (16) become significant.

The implementation of Kriging-DIC in this paper is as follows: Firstly a Kriging model of the displacement field in the RoI is formulated using a certain number of artificially selected control points in the reference image. A fast DIC subset method [21] was adopted to obtain approximate displacements for the control points. This fast 
DIC method employs square subsets of identical size in the reference and deformed images respectively to calculate the correlation coefficients between them. The selected control points are the centre points of the subsets in the reference image. When the matched subsets (having maximum correlation) are found, the initial displacements of control points are achieved as the differences between centre points of matched subsets. This method will generally obtain the integer-pixel displacements accurately to within 3 pixels, based on empirical evidence, which is close enough for initial values of Kriging-DIC method. Vendroux and Knauss [35] showed that the Newton iteration method has a convergence radius of 7 pixels for initial values. Zhao et al. [37] introduced a number of strategies to improve the robustness of DIC solutions to variability in initial estimates of displacements, especially for cases of large deformation.

Secondly, an updating procedure can be applied to add more control points into the initial Kriging model to achieve a more suitable model. This updating procedure will be introduced in the following section. It should be noted that this updating procedure is not necessary if sufficient control points are artificially selected using a fixed control grid.

Finally, on achieving a suitable Kriging model, Newton iteration is applied to obtain the final displacement field with the sub-pixel accuracy in the inversion of Equation (3). Moreover, as the grey values of non-integer pixels are required in this process, a grey-value interpolation scheme is needed and for reasons of simplicity a $6 \times 6$ bicubic interpolation scheme was chosen work the examples presented in this study.

\section{Self-adaptive Control Grid Updating}

For DIC problems, it is desirable to have an algorithm that determines an optimal control grid. Generally, a finer control grid does not guarantee a more accurate measurement. However, a fine control grid is necessarily applied in the case of a complex deformation, when a coarse control grid would fail to capture the detail of local deformations.

Kriging provides the error estimations over the whole RoI and therefore it is necessary to improve the control grid by adding new sample points. The estimated Mean Squared Error (MSE) of the Kriging model provides a criterion for achieving such an improvement. The MSE at any location $(x, y)$ may be expressed as [38],

$$
\varepsilon(x, y)=\hat{\sigma}^{2}\left(1+\boldsymbol{\tau}^{T}\left(\mathbf{C}^{T} \mathbf{R}^{-1} \mathbf{C}\right)^{-1} \boldsymbol{\tau}-\mathbf{r}^{T}(x, y) \mathbf{R}^{-1} \mathbf{r}(x, y)\right)
$$

where $\boldsymbol{\tau}=\mathbf{C}^{\mathrm{T}} \mathbf{R}^{-1} \mathbf{r}(\mathbf{x})-\mathbf{c}(\mathbf{x})$, and $\hat{\sigma}^{2}$ is given from Equation (13) with $\hat{\boldsymbol{\beta}}$ from (14). It should be noted that adding new points imposes a compromise between accuracy and numerical stability. Even if the estimation improves due to the presence of more 
information carried by the data, having too many points may cause the columns of $\mathbf{R}$ to become numerically close and therefore linearly dependent for practical purposes.

As has been already stated, Kriging regression allows for the effect of measurement error at the sample points, determined according to the optimised term $e_{M}^{2}$. Of course this error is fully justified and has nothing to do with the lack of knowledge represented by the Gaussian process present in the Kriging model, which we seek to reduce by adding new sample points at candidate locations where the MSE is greatest. The problem is that Equation (17) does not discriminate between the measurement error and lack of knowledge, and this inevitably leads to dense clusters of added points very close to the original control points and to stalling of progress towards the desired Kriging model [26, 29]. This can be overcome by reformulation of the Kriging model as an interpolator (rather than a regressor) with the control-point error added to the coordinates of each control point.

Thus the infill criterion in the present study is the Maximum Mean Square Error (MMSE). An alternative infill criterion was proposed by Forrester et al. [26, 29, 39] in which the objective function is the Kriging output with a minimum at an unknown coordinate. This enables added control point to be chosen that have the greatest effect on reducing the objective function. In the present case a constrained global objective function is defined by equation (1) and Forrester's criterion is therefore not applicable. This means that selecting a new control point based on the local MMSE does not necessarily lead to a reduction of the objective function. The Global Mean Square Error (GMSE), defined as the mean of the calculated MSE function over the region of interest, is used as the stopping criterion for control-grid updating.

The updating process can be briefly summarised as follows.

(i) Control points are selected on the edges of the RoI of the reference image, the

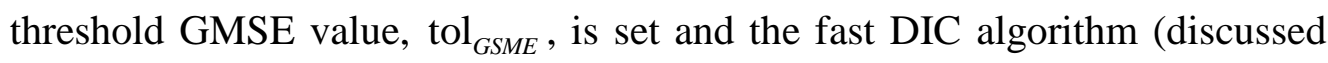
previously) is used to obtain an approximate measurement of the control-point displacements (The choice of the points on the edges of the RoI is made due to a well-known property of Kriging: whilst it delivers reliable interpolation given observed data, and such interpolation improves as more observations become available, it can perform poorly when extrapolating for training runs which are not in the neighbourhood of the available data samples).

(ii) The Kriging regression model (10) is constructed (including optimised $e_{M}^{2}$ ) and displacements determined using Equation (8).

(iii) Construct a new Kriging model with the measurement error $e_{M}^{2}$ from (ii) added to the control point coordinates. Then set $e_{M}^{2}=0$ and the new model becomes a Kriging interpolator. Compute the MSE and GMSE.

(iv) GMSE $<\operatorname{tol}_{G M S E}$ ? If not, add new control points at coordinates of greatest MSE and return to step (ii). If so, the Kriging model is complete. 


\section{Case Studies}

Three case studies are included to illustrate the performance of Kriging-DIC. In the first of these, experimental speckle-pattern images from the DIC Challenge 2D Dataset [40] are translated by 2.2 and 3.3 pixels in the $x$ - and $y$-directions respectively. Kriging-DIC results are compared to those obtained by Q4-FE DIC and Cubic-Spline DIC methods. The second case study concerns a numerically-produced complex displacement field using grey-scale images generated by (a) interpolation from the FE model and (b) Gaussian speckles on the reference and deflected images. Finally, application of Kriging-DIC is demonstrated in an experimental example.

\section{Case Study 1: DIC Challenge Data-Rigid body displacement.}

Experimental speckle pattern images from DIC Challenge 2D Dataset [40] were translated by 2.2 and 3.3 pixels in the $\mathrm{x}$ - and y-directions respectively by Fourier transformation [21, 41], achieved by phase shifts without change of amplitude. The RoI, spanning $101 \times 101$ pixels, is uniformly meshed by $5 \times 5$ square elements, each of $21 \times 21$ pixels. This grid provides $6 \times 6$ control points (nodes) so that the full-field displacement is determined by the displacement of 36 control points as shown in Figure 2.

The displacement field is calculated by three different full-field DIC methods, Kriging-DIC, Q4-FE DIC [4, 5, 20] and Cubic Spline DIC [14, 15] using B-spline basis functions. To ensure comparability, the number and location of the control points are fixed so that control-grid updating is not applied in the Kriging-DIC approach. Kriging regression was applied, but in this particular example it was found that $e_{M}^{2}=0$, identical to the case of Kriging interpolation. This is to be expected since the true displacements of all the control points are the same (2.2 in $x$-direction, 3.3 in $y$-direction) and the initial displacements of all the control points calculated by the fast method are the same as well ( 2 in $x$-direction, 3 in $y$-direction). The number of degrees of freedom are the same for each of the three approaches.

The initial displacements of the 36 control points were obtained by the fast DIC method based on integer pixels and 20 Newton iterations were subsequently carried out using 6x6 point bi-cubic interpolation [42] for sub-pixel grey values. Results are summarised in Table 1 and Figure 3 and 4 where it can be seen that the displacement field produced by Kriging DIC is smoother than that produced by Q4-FE DIC, which shows some significant ridges at the element boundaries. The Kriging results are also better than those produced Cubic Spline DIC, which shows some abrupt peaks and greater deviations than Kriging DIC. Kriging is seen to produce a full-field measurement with lower mean error and standard deviation (STD) than the other two methods. The small biases shown in Figure 3 and 4 are due to the effect of bi-cubic grey-scale interpolation [21, 41, 43]. 


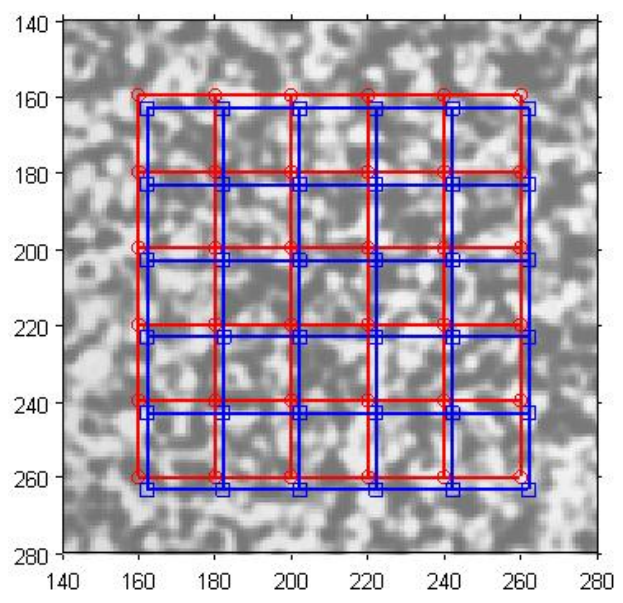

Figure 2 Reference and deformed grids shown as red and blue squares respectively.

Table 1 Errors Comparison (unit pixel)

\begin{tabular}{|c|c|c|c|c|}
\hline \multicolumn{2}{|c|}{} & Kriging & Q4-FE & Cubic Spline \\
\hline \multirow{2}{*}{$\mathrm{X}$} & Mean Error & $5.44 \mathrm{e}-3$ & $8.10 \mathrm{e}-3$ & $5.72 \mathrm{e}-3$ \\
\cline { 2 - 5 } & STD & $1.23 \mathrm{e}-3$ & $1.57 \mathrm{e}-3$ & $1.33 \mathrm{e}-3$ \\
\hline \multirow{2}{*}{$\mathrm{Y}$} & Mean Error & $3.34 \mathrm{e}-3$ & $4.98 \mathrm{e}-3$ & $3.74 \mathrm{e}-3$ \\
\cline { 2 - 5 } & STD & $1.04 \mathrm{e}-3$ & $1.46 \mathrm{e}-3$ & $1.50 \mathrm{e}-3$ \\
\hline
\end{tabular}

* Mean Error here is the difference between actual Mean and the theoritical values i.e. $2.2 \& 3.3$ pixels
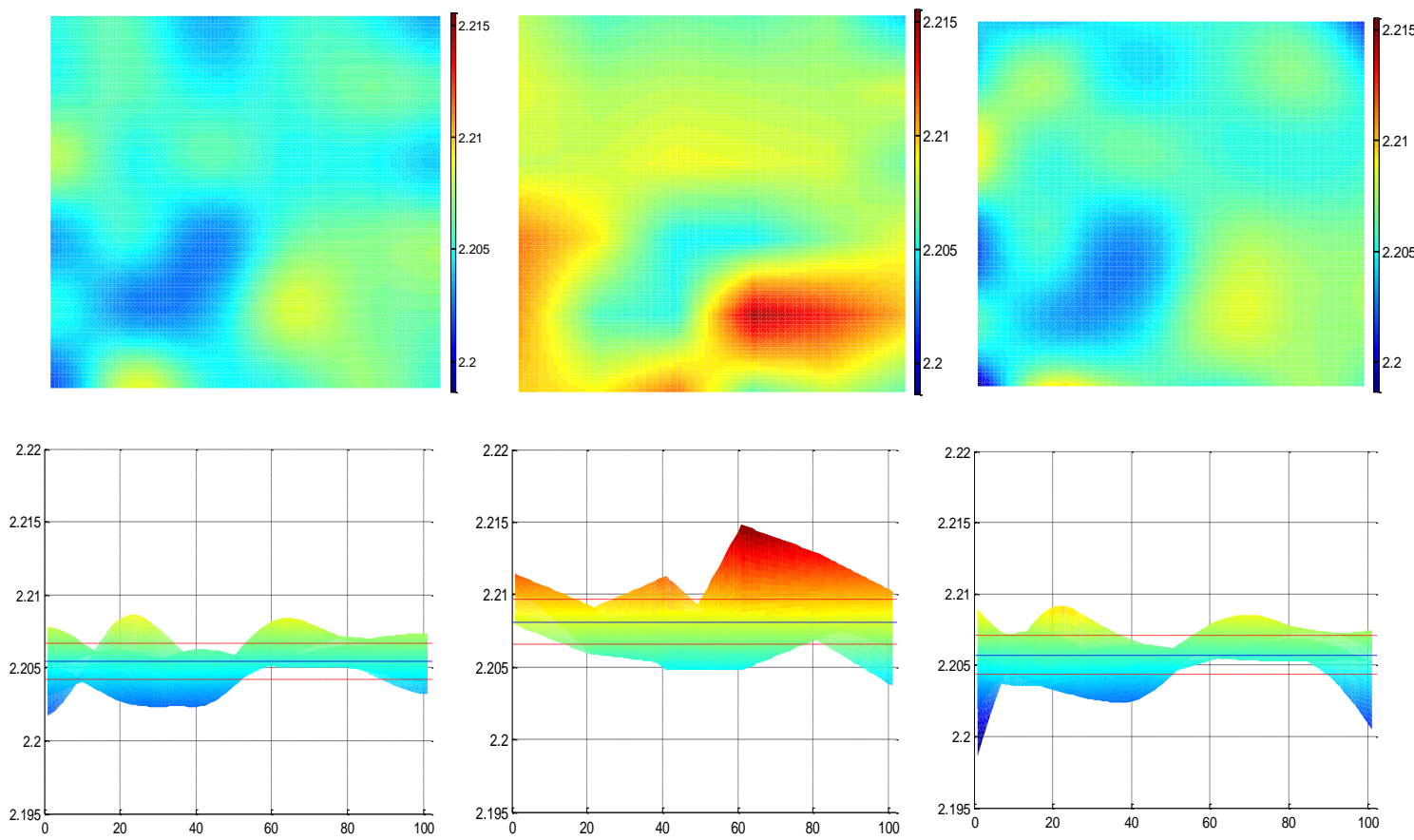

Figure 3 Calculated displacement fields in X direction (real displacement 2.2 pixels), from left to right: Kriging DIC, Q4-FE DIC and Cubic Spline DIC, and '--' indicates the Mean while '-.' indicates the Standard Deviation. 

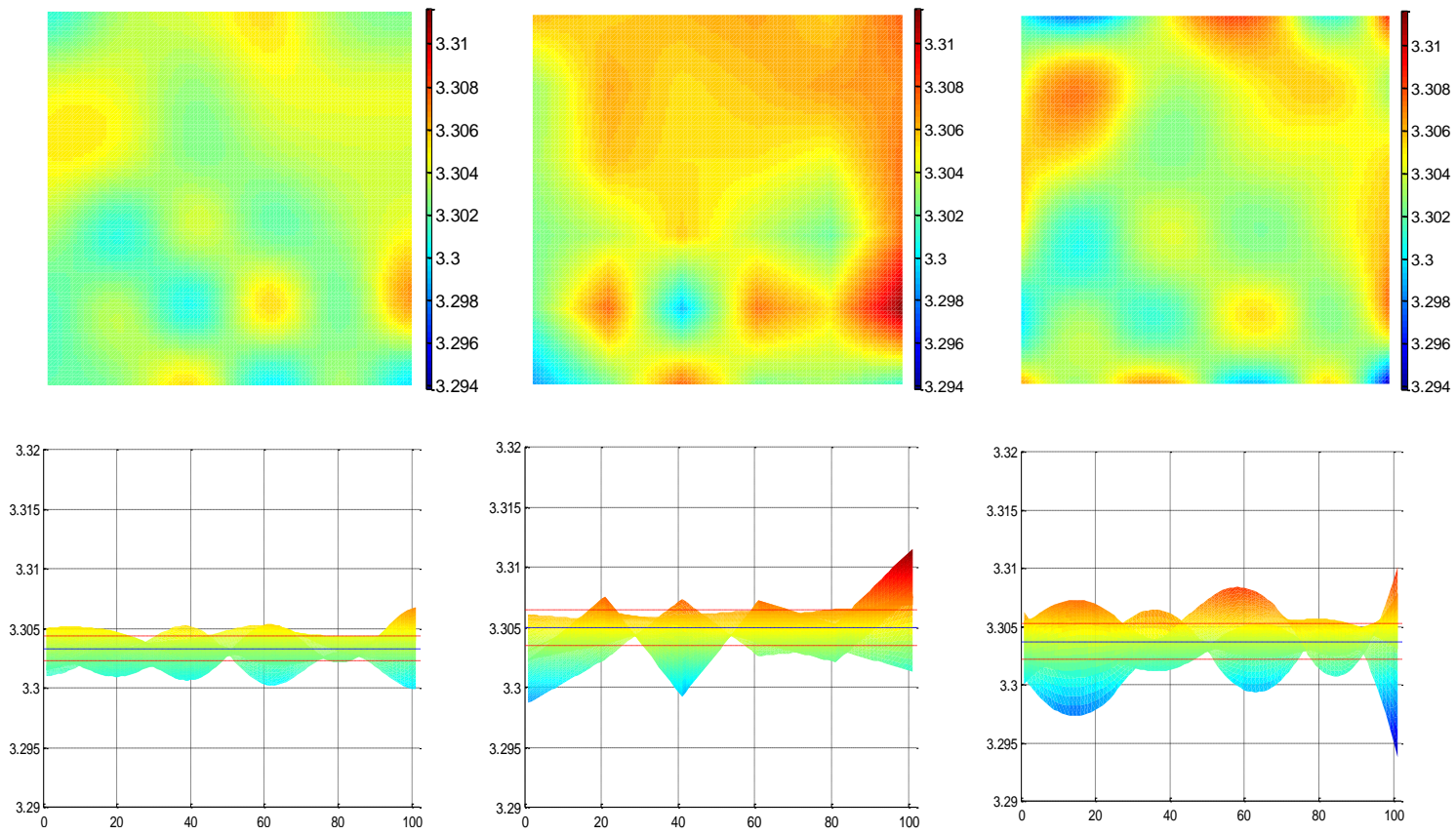

Figure 4 Calculated displacement fields in Y direction (real displacement 3.3 pixels, from left to right: Kriging DIC, Q4-FE DIC and Cubic Spline DIC, and '--' indicates the Mean while '-.' indicates the Standard Deviation

Case study 2: Non-uniform displacement field with numerically produced speckles.

In this case study, two examples having same displacement field but using numerically-produced grey-scale images generated by different methods are presented. The displacement field is calculated from a FE model and used to test the performance of the Kriging-DIC method with control grid updating.

In the first approach, displacements at integer pixel locations are determined by FE shape-function interpolation. The deformed image is then generated by displacing the speckle pattern (DIC Challenge 2D Dataset [40]) of the reference image pixel-bypixel by the corresponding FE displacement. The second approach is based on numerically-produced Gaussian speckles [44, 45] (not directly related to the Gaussian process that forms part of the Kriging model) with means uniformly distributed over the RoI. In the present case 7000 independent and identically distributed speckles are superimposed on the raw image of $250 \times 300$ pixels, each Gaussian speckle having a standard deviation of 2.5 pixels. The speckles of the deformed image are obtained by shifting the means (of the reference-image speckles) by the displacements determined from the FE model. The reference and deformed images are digitised using an 8-bit processor. Finally, in both cases, bi-cubic interpolation is applied to determine the grey-scale images.

The FE model of the square plate $(100 \times 100 \times 10 \mathrm{~mm})$ in steel, with a central hole of radius of $20 \mathrm{~mm}$, is composed of a very fine mesh of CQUAD4 elements with a total of 10,400 nodes. The RoI consists of $250 \times 250$ pixels and for DIC the reference and deformed images are trimmed out along the outer edges and around the edge of the 
hole to avoid edge effects. The FE displacement field in the $x$ - and $y$-directions are shown in Figure 5 when the left hand side of the plate is encastre and a uniformly distributed tensile load on the right-hand edge produces an elastic extension of approximately $14 \mathrm{~mm}$. Initial control points were selected close to the edges of the RoI denoted by the red '+' signs in Figure 6. The control grid was updated adaptively as described in Section 3 using the fast DIC method (described previously). At each grid updating step two new control points were added having the greatest MSE in the $x$ - and $y$-directions. Figure 6 shows the added control points as blue ' $x$ ' signs for the case of the first approach based on FE interpolation. Updating was continued until the GMSE was deemed sufficiently small according to a pre-set tolerance.
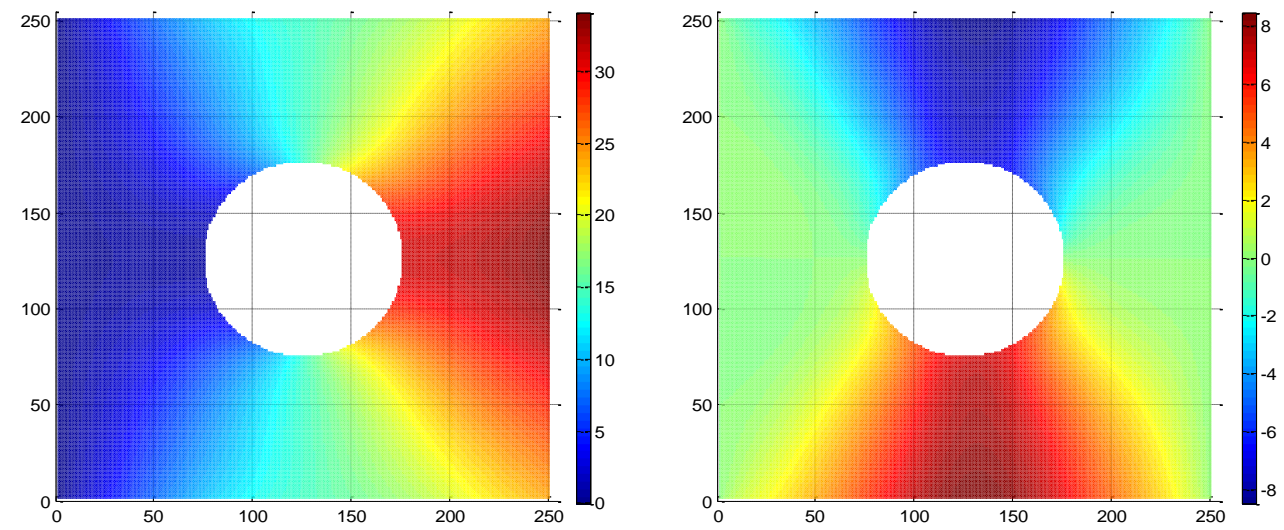

Figure 5 The interpolated FE displacement fields in $x$-direction (left) and $y$-direction (right)
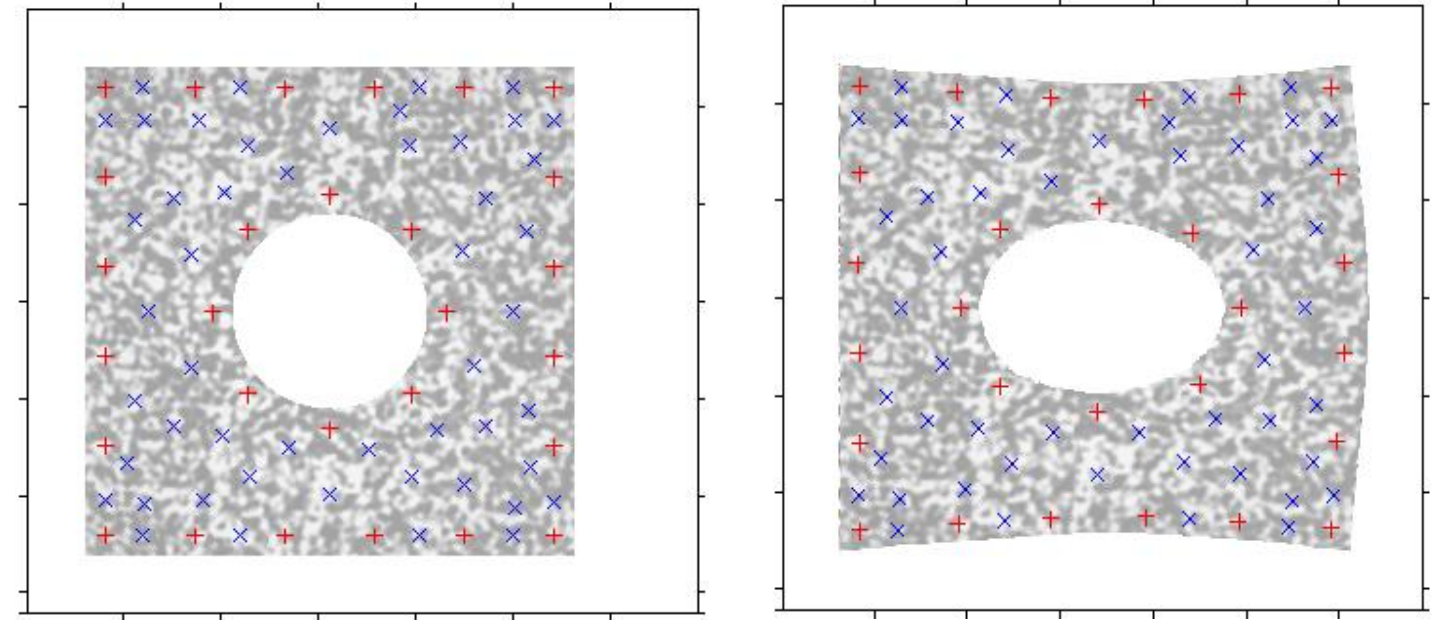

Figure 6 The distribution of 78 chosen control points (28 initial points) on the reference image (left) and the deformed image (right).

Results from the both approaches are shown in Figure 7-12. The evolution of GMSE is shown in Figure 7 and optimisation parameters $\vartheta_{x}, \vartheta_{y}$ and $e_{M}^{2}$ in Figure 8. Figure 7 and 8 show results obtained from the first approach with the 28 initial control points in Figure 6 and also from a different initial condition of 16 control points arranged irregularly around the boundary. The fully converged Kriging model has 78 control 
points (approach 1, both 28 and 16 initial points) and 88 control points (approach 2) after adaptive control-grid updating. The objective function in Figure 9 is normalised by the sum of all the grey intensities in the reference image and is therefore unit-less. The error in the $x$ - and $y$-displacement fields is shown in Figure 10 and 11 for the two approaches and the evolution of the mean error and standard deviation is given in Figure 12.

The optimisation parameters $\vartheta_{x}$ and $\vartheta_{y}$ from the two approaches were found to be similar, although there were differences in the values of $e_{M}^{2}$, presumably due to the different speckle patterns produced by the two methods. Convergence of the objective function was somewhat slower by the second approach, but the final estimated displacement fields were found to be almost identical from visual inspection of Figure 10 and 11. The statistics of displacement fields are given in Table 2. The displacement fields from approach 1 were found to converge to almost identical statistics regardless of the number and location of the initial control points.

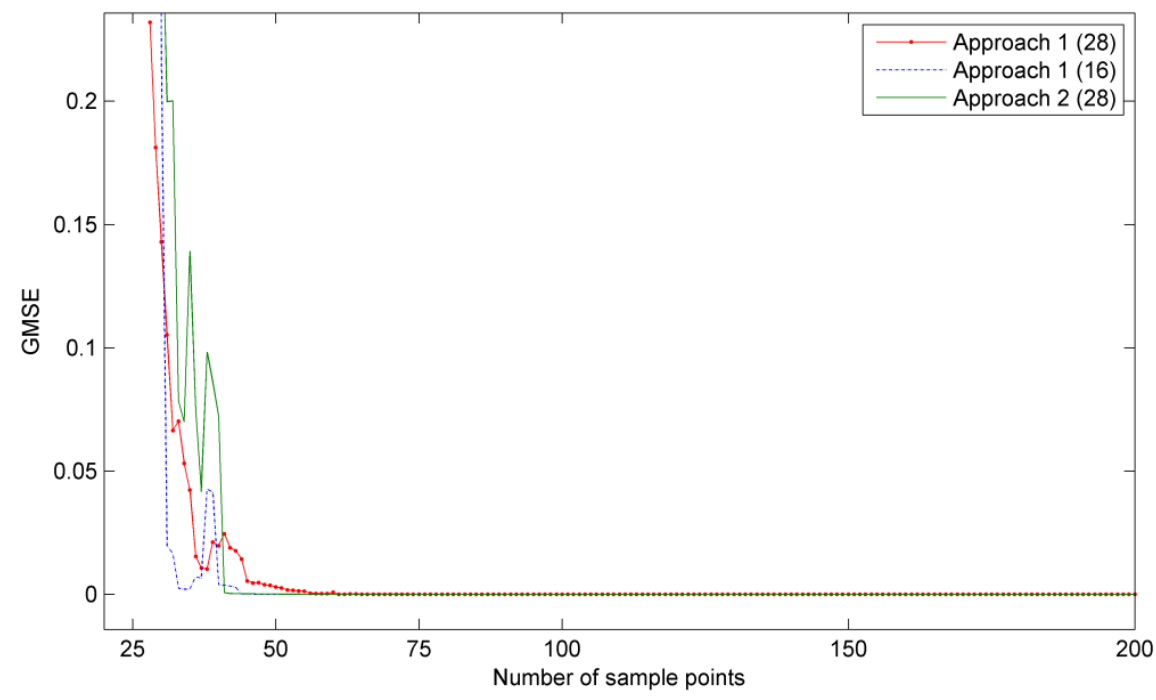

Figure 7 Evolution of GMSE: Approach 1 with 28 \& 16 initial control points; Approach 2 with 28 initial points.

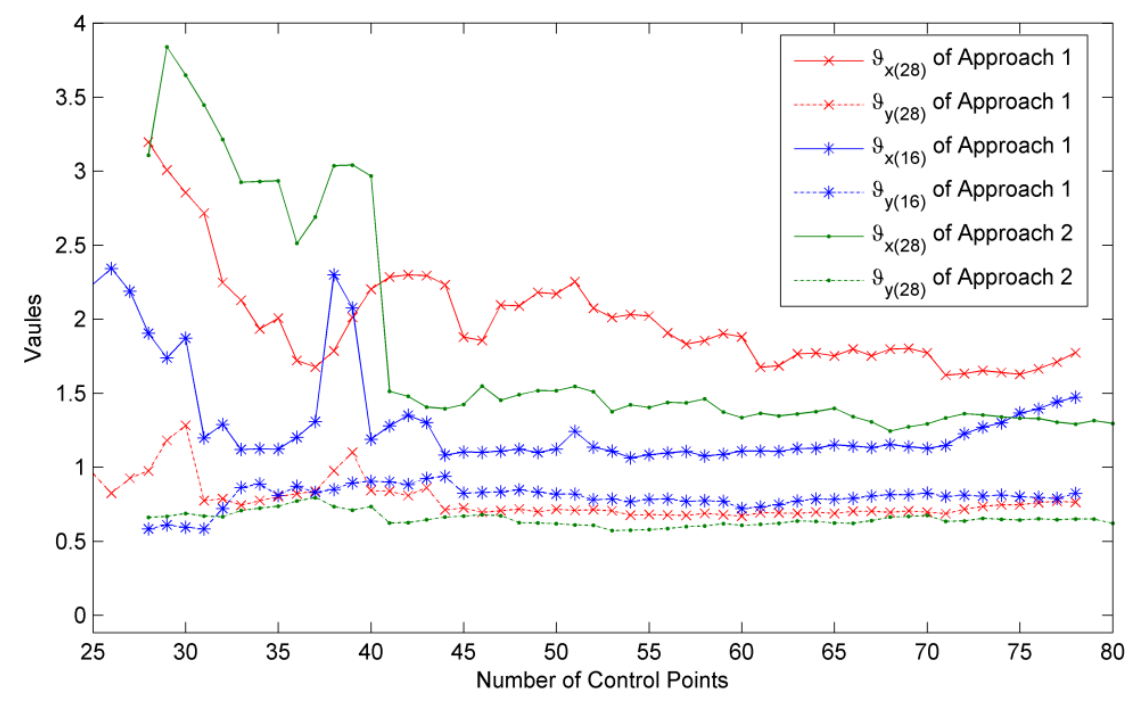




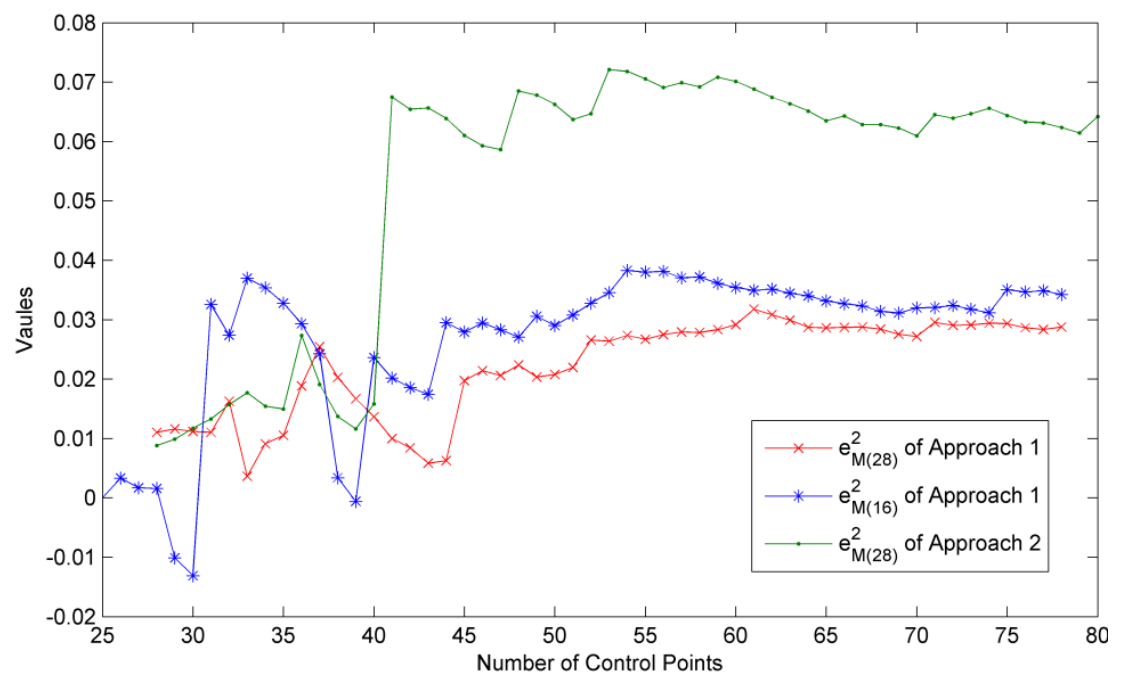

Figure 8 Evolution of optimization parameters with increasing numbers of control points.

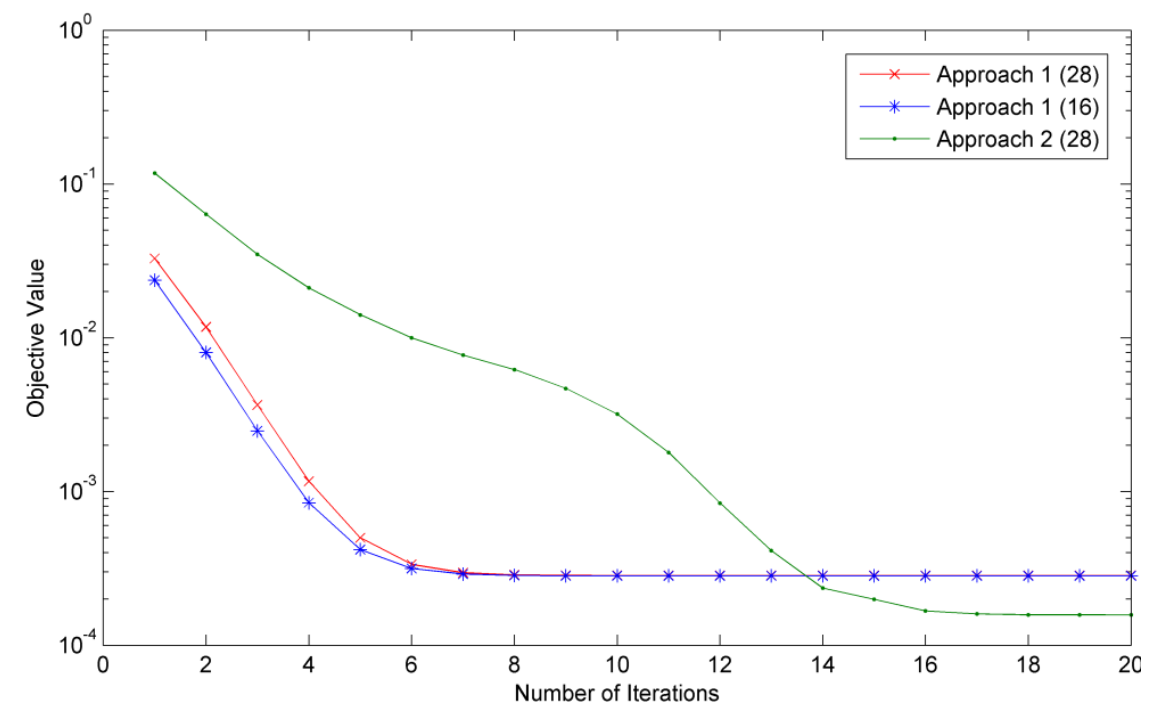

Figure 9 Convergence of the objective function - Newton iteration.
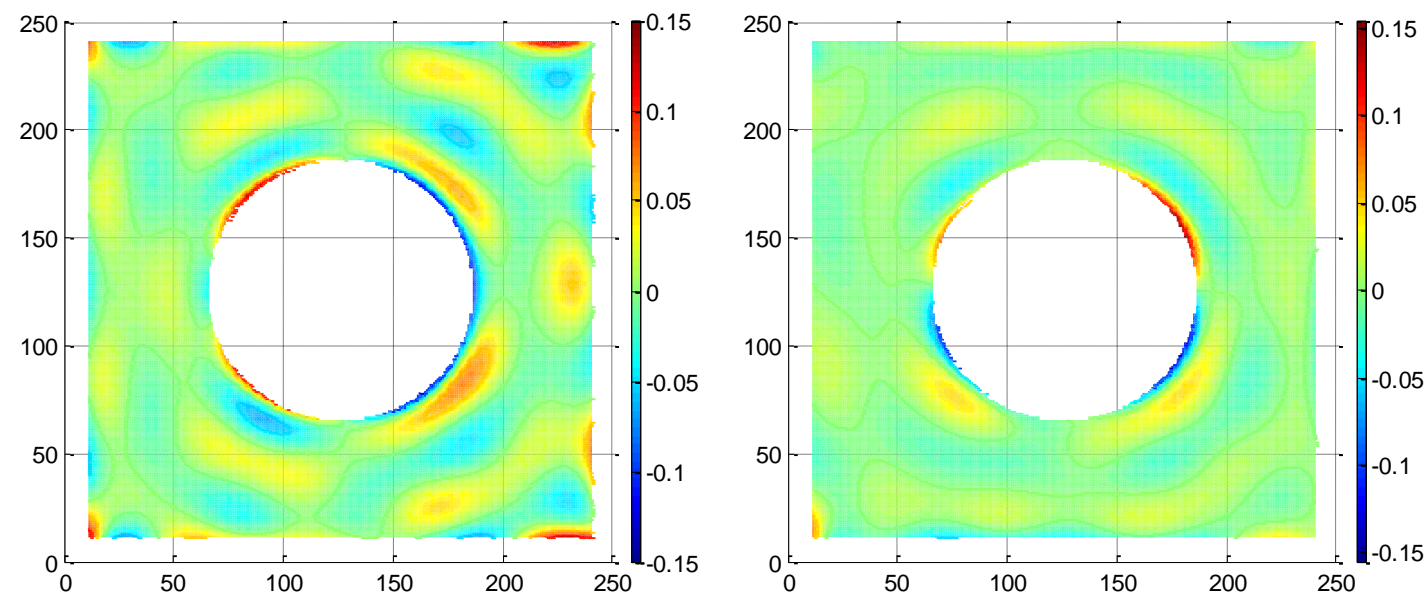

Figure 10 Displacement errors in pixels (first approach, 28 initial points) after Newton iteration in $x$-direction (left) and $y$-direction (right) 

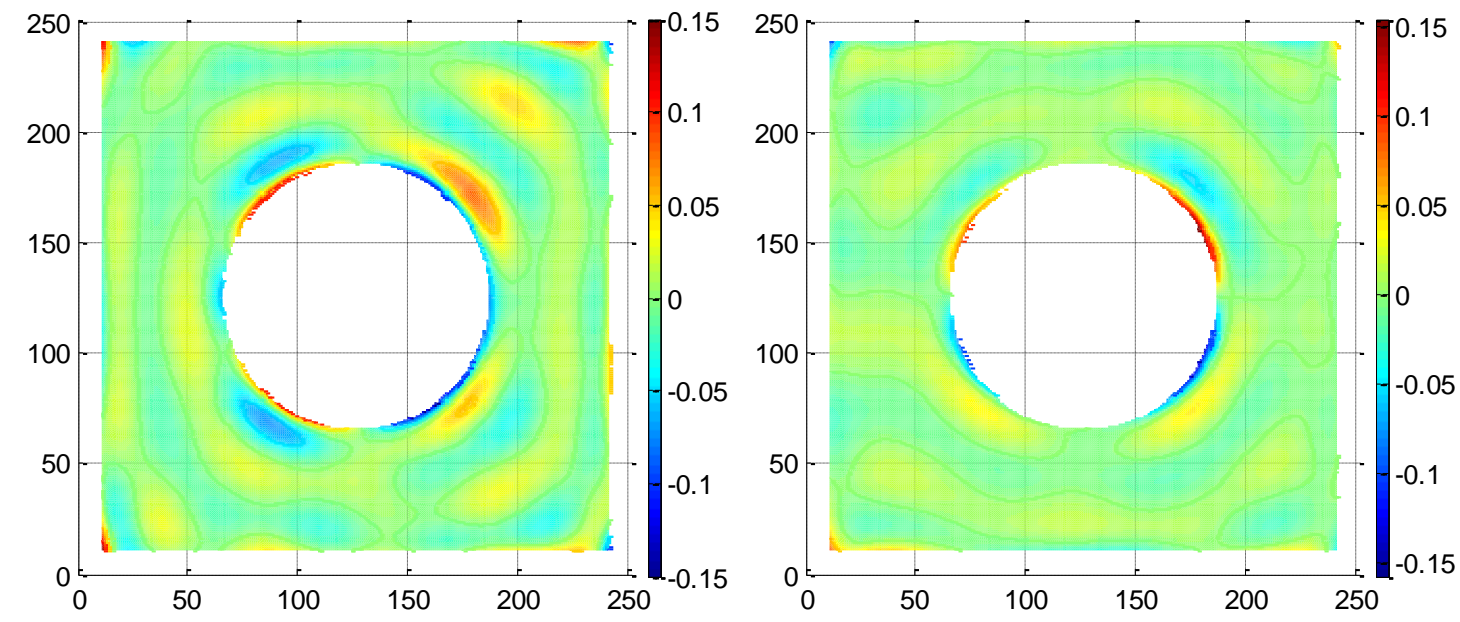

Figure 11 Displacement errors in pixels (second approach, 28 initial points) after Newton iteration in $x$-direction (left) and $y$-direction (right)
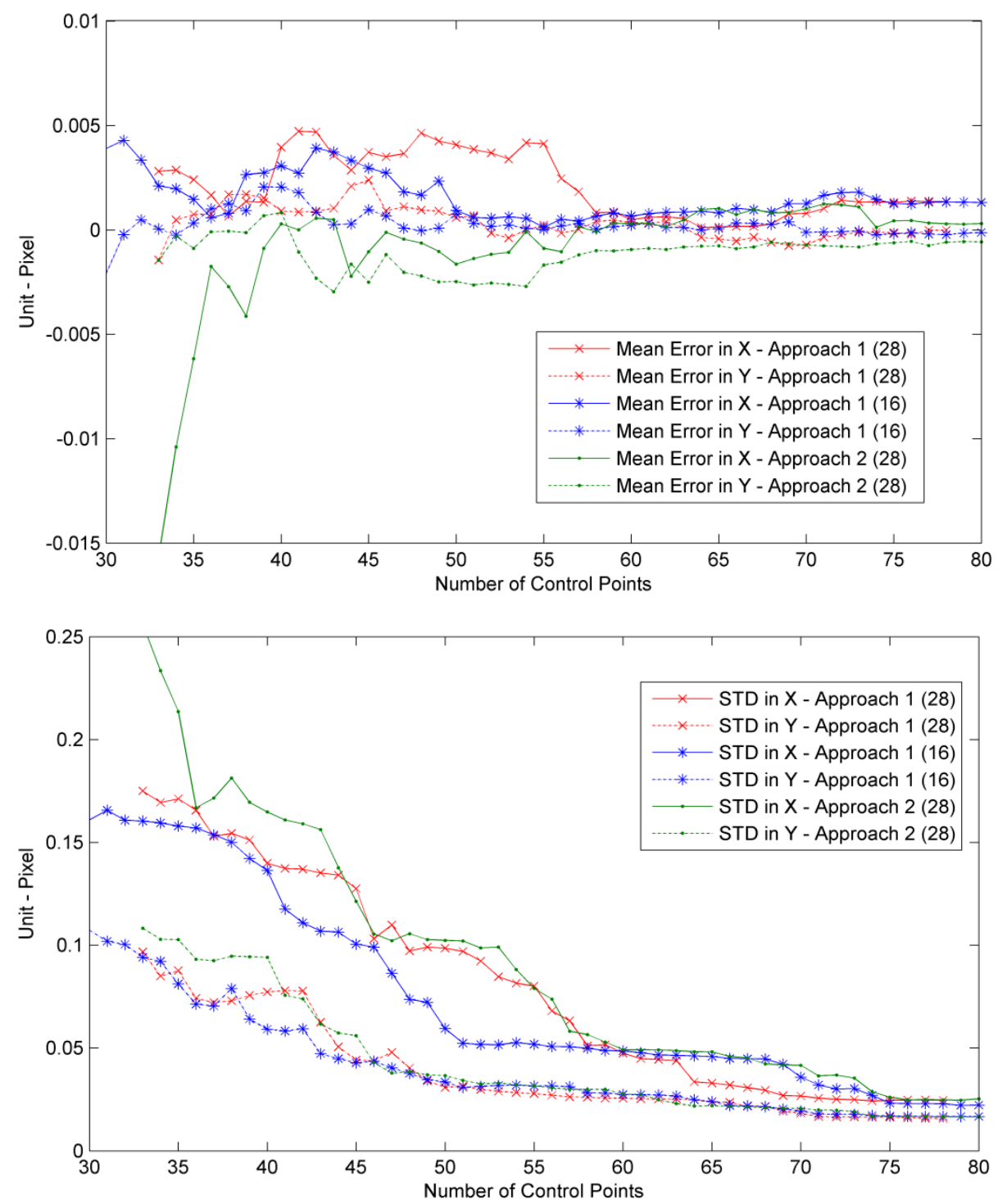

Figure 12 Evolution of Kriging-DIC measurement error statistics 
Table 2 Measurement-error statistics in pixels.

\begin{tabular}{|c|c|c|c|c|}
\hline \multicolumn{2}{|c|}{} & $\begin{array}{c}\text { Approach 1 } \\
\text { (28 initial points \& 78 } \\
\text { in total) }\end{array}$ & $\begin{array}{c}\text { Approach 1 } \\
\text { (16 initial points \& 78 } \\
\text { in total) }\end{array}$ & $\begin{array}{c}\text { Approach 2 } \\
\text { (28 initial points \& 88 } \\
\text { in total) }\end{array}$ \\
\hline \multirow{3}{*}{ X } & $\begin{array}{c}\text { Mean } \\
\text { Error }\end{array}$ & 0.00135 & 0.00134 & $2.60 \mathrm{e}-4$ \\
\cline { 2 - 5 } & STD & 0.0244 & 0.0228 & 0.0209 \\
\hline \multirow{3}{*}{ Y } & $\begin{array}{c}\text { Mean } \\
\text { Error }\end{array}$ & $-1.51 \mathrm{e}-5$ & $-2.25 \mathrm{e}-4$ & $-4.93 \mathrm{e}-4$ \\
\cline { 2 - 5 } & STD & 0.0157 & 0.0166 & 0.0151 \\
\hline
\end{tabular}

\section{Case Study 3: Experimental I-beam test}

Application of the Kriging-DIC method is demonstrated on an experimental I-section beam with circular holes arranged symmetrically along the beam about its centre as shown in Figure 13. The overall dimensions of the cross section are $42 \mathrm{~mm} \times 65 \mathrm{~mm}$ with $2.5 \mathrm{~mm}$ wall thickness. The distance between the supports is $450 \mathrm{~mm}$. The test arrangement shown in the figure is designed to apply a mid-span transverse point load, in the present case $2 \mathrm{kN}$. The experimental setup is described in detail by Labeas et al. [46].

The speckle-pattern reference image for a square RoI of $700 \times 700$ pixels is shown in Figure 14 with 12 initial control points denoted by red ' + ' signs and 30 added control points shown by blue ' $x$ ' signs. The estimated displacement field determined by Newton iteration is shown in Figure 15 and for purpose of comparison results from a commercial system using a local, subset-based DIC approach $(41 \times 41$ pixel subsets and 30 pixel grid spacing) is provided in Figure 16. Figure 17 shows the absolute difference between the Kriging-DIC result and that produced by the commercial system with the statistics of the difference summarised in Table 3. Results from the two systems appear to be similar although the displacement field produced by Kriging DIC seems smoother than that produced by the commercial system, which shows unexpected oscillations in the y-direction displacement field (Figure 16).

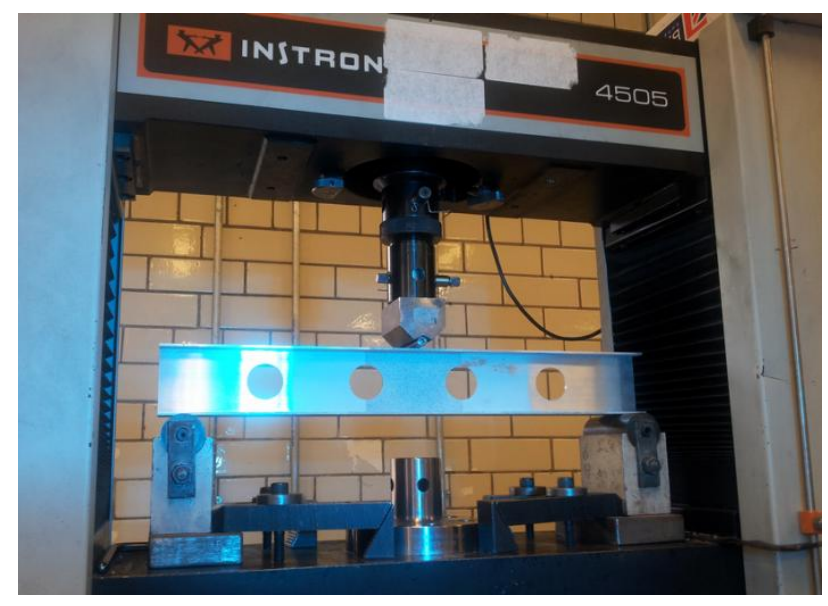

Figure 13 The experimental setup. 


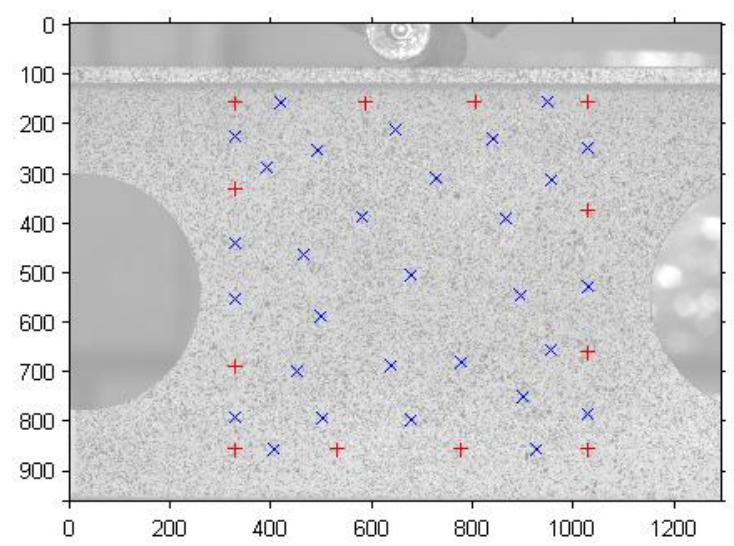

Figure 14 The distribution of initial control points (red ' + ' signs) and added control points (blue ' $\times$ ' signs) superimposed on the reference image.
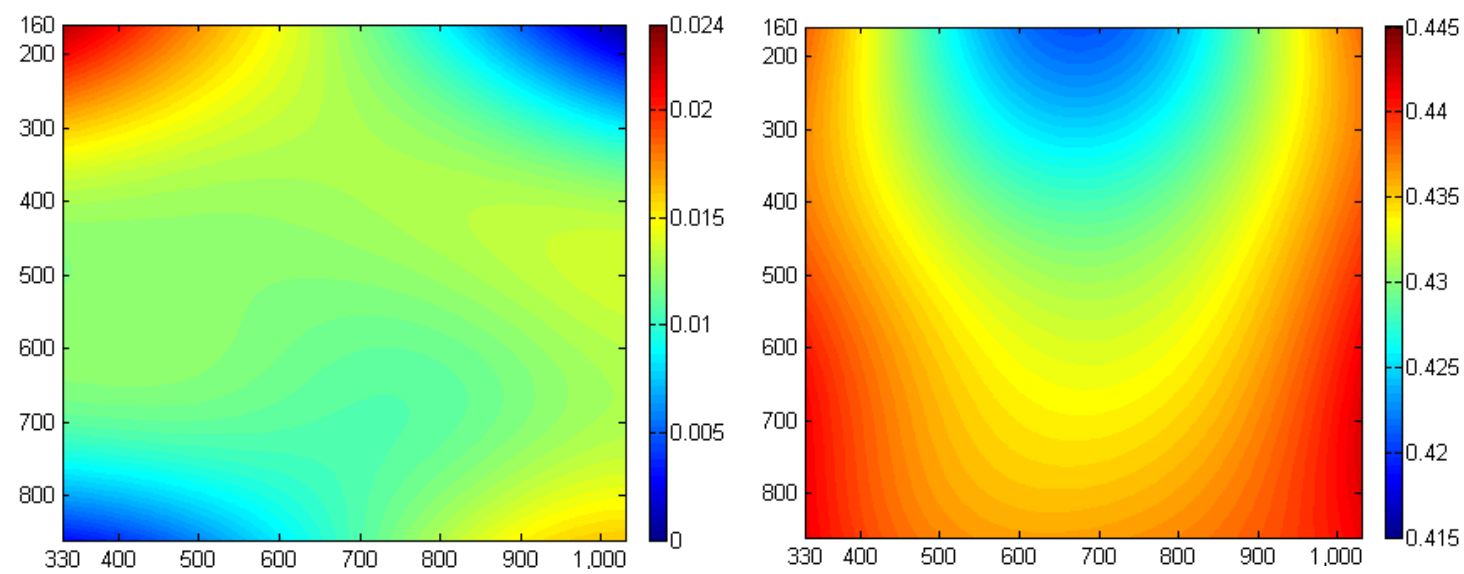

Figure 15 Displacement fields $(\mathrm{mm})$ calculated by Kriging DIC method in $x$-direction (left) and $y$-direction (right).
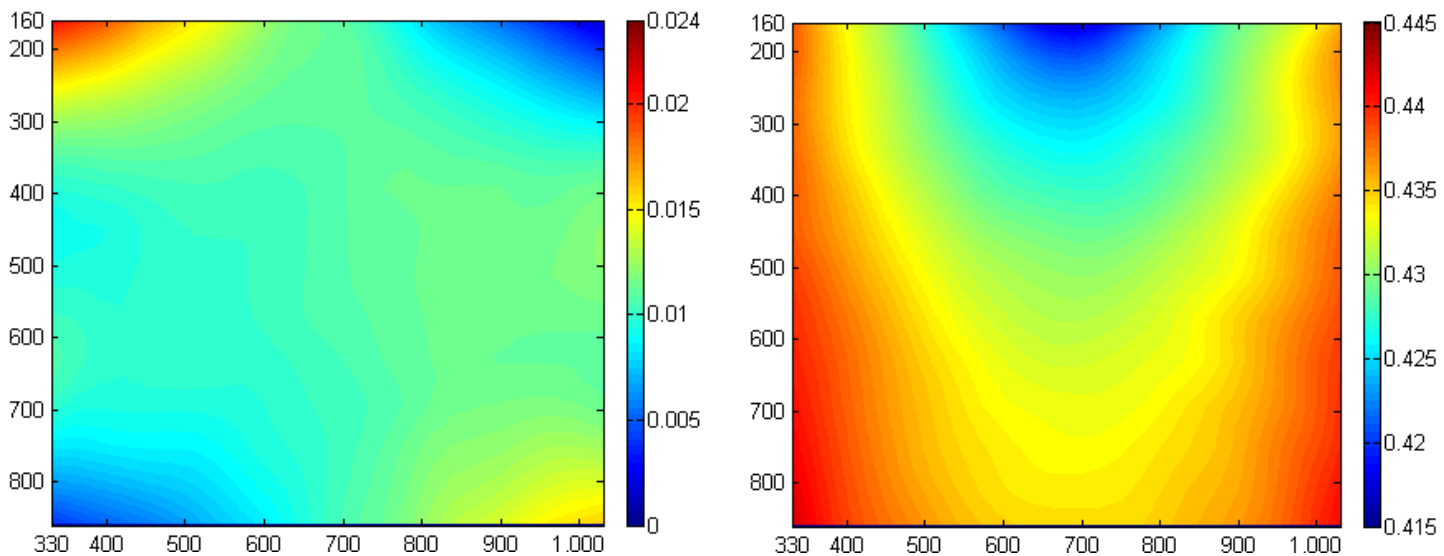

Figure 16 Displacement fields (mm) calculated by the commercial system in the $x$ direction (left) and $y$-direction (right) 

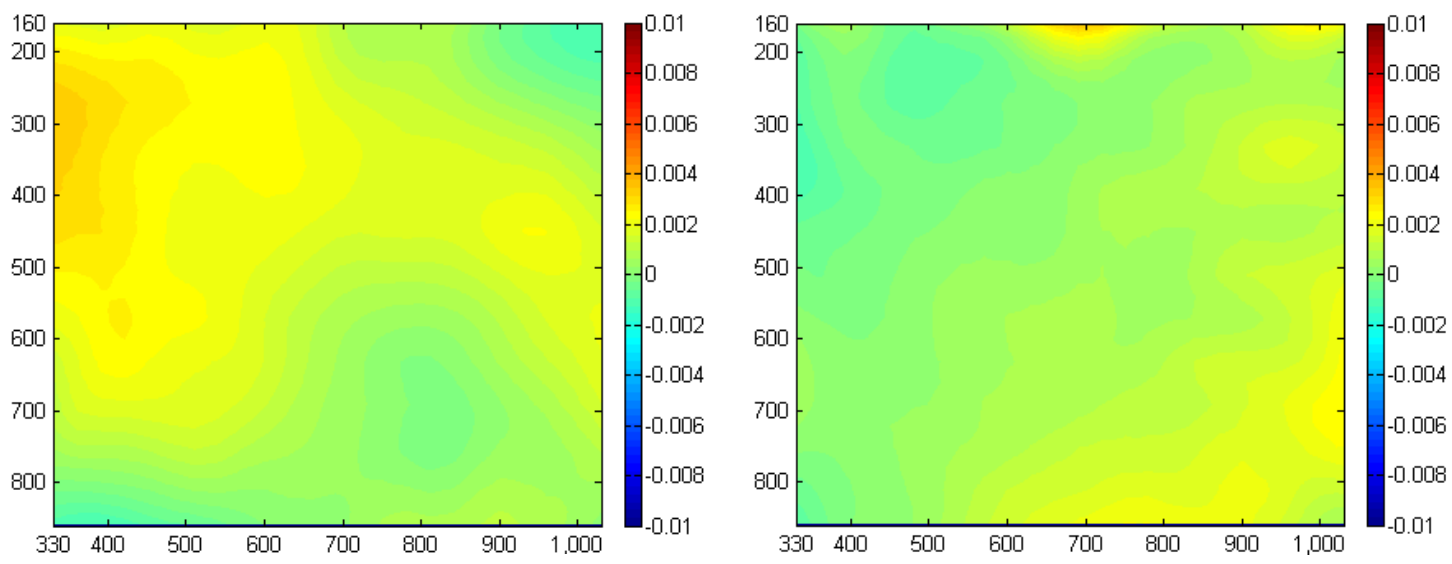

Figure 17 The absolute difference between the displacement fields ( $\mathrm{mm}$ ) calculated by Kriging DIC and the commercial system in the $x$-direction (left) and $y$-direction (right).

Table 3 Mean values and standard deviations of the absolute difference

\begin{tabular}{|c|c|c|c|}
\hline \multicolumn{2}{|c|}{ Residual Disp } & Unit: $\mathrm{mm}$ & Unit: pixel* \\
\hline \multirow{2}{*}{$x$} & Mean & $1.3472 \mathrm{e}-3$ & 0.0182 \\
\cline { 2 - 4 } & STD & $9.1654 \mathrm{e}-4$ & 0.0124 \\
\hline \multirow{2}{*}{$y$} & Mean & $6.0095 \mathrm{e}-4$ & 0.0081 \\
\cline { 2 - 4 } & STD & $7.3189 \mathrm{e}-4$ & 0.0099 \\
\hline
\end{tabular}

*1 pixel length $\approx 0.074 \mathrm{~mm}$

\section{Conclusions}

A DIC method based on Kriging regression with self-adaptive control grid updating is developed. The Kriging approach consists of two parts based on a regression model and the correlation between displacements at control points. The result is a minimum variance estimator with the error represented by a Gaussian process. The control grid may be updated self-adaptively using the mean square error determined at locations away from the control points. Unlike Q4-FE DIC and Cubic-Spline DIC, Kriging DIC is based on control points that are distributed fully throughout the region of interest. The methodology is supported by three case studies, the first of which uses experimental data from the DIC Challenge 2D database. Using this data Kriging-DIC is shown to outperform Q4-FE DIC and Cubic Spline DIC. In the second case study Kriging DIC is shown to be robust to the number and location of initially-chosen control points and to speckle-pattern variation. The third case study is an experimental example where Kriging DIC is shown to perform favourably against a commercial subset-based DIC system. 


\section{References}

1. Gates, M., Lambros, J., and Heath, M.T., Towards High Performance Digital Volume Correlation. Experimental Mechanics, 2011. 51(4): p. 491-507.

2. Zhao, J.Q., et al., Improved Hermite finite element smoothing method for full-field strain measurement over arbitrary region of interest in digital image correlation. Optics and Lasers in Engineering, 2012. 50(11): p. 1662-1671.

3. Mortazavi, F., Development of a global digital image correlation approach for fast high-resolution displacement measurements, in Department of Mechanical Engineering. 2013, École Polytechnique de Montréal, : Montreal.

4. Sun, Y.F., et al., Finite element formulation for a digital image correlation method. Applied Optics, 2005. 44(34): p. 7357-7363.

5. Besnard, G., Hild, F., and Roux, S., "Finite-Element" displacement fields analysis from digital images: Application to portevin-le chatelier bands. Experimental Mechanics, 2006. 46(6): p. 789-803.

6. Passieux, J.C. and Perie, J.N., High resolution digital image correlation using proper generalized decomposition: PGD-DIC. International Journal for Numerical Methods in Engineering, 2012. 92(6): p. 531-550.

7. Avril, S., et al., Overview of identification methods of mechanical parameters based on full-field measurements. Experimental Mechanics, 2008. 48(4): p. 381-402.

8. Leclerc, H., et al., Integrated Digital Image Correlation for the Identification of Mechanical Properties. Computer Vision/Computer Graphics Collaboration Techniques, Proceedings, 2009. 5496: p. 161-171.

9. Langerholc, M., Slavic, J., and Boltezar, M., Absolute Nodal Coordinates in Digital Image Correlation. Experimental Mechanics, 2013. 53(5): p. 807-818.

10. Moes, N., Dolbow, J., and Belytschko, T., A finite element method for crack growth without remeshing. International Journal for Numerical Methods in Engineering, 1999. 46(1): p. 131-150.

11. Rethore, J., et al., Estimation of mixed-mode stress intensity factors using digital image correlation and an interaction integral. International Journal of Fracture, 2005. 132(1): p. 65-79.

12. Roux, S. and Hild, F., Stress intensity factor measurements from digital image correlation: post-processing and integrated approaches. International Journal of Fracture, 2006. 140(1-4): p. 141-157.

13. Rethore, J., Hild, F., and Roux, S., Extended digital image correlation with crack shape optimization. International Journal for Numerical Methods in Engineering, 2008. 73(2): p. 248-272.

14. Cheng, P., et al., Full-field speckle pattern image correlation with B-spline deformation function. Experimental Mechanics, 2002. 42(3): p. 344-352.

15. Rethore, J., et al., On the Use of NURBS Functions for Displacement Derivatives Measurement by Digital Image Correlation. Experimental Mechanics, 2010. 50(7): $p$. 1099-1116.

16. Mortazavi, F., Levesque, M., and Villemure, I., Image-based Continuous Displacement Measurements Using an Improved Spectral Approach. Strain, 2013. 49(3): p. 233-248.

17. Roux, S., Hild, F., and Berthaud, Y., Correlation image velocimetry: a spectral approach. Applied Optics, 2002. 41(1): p. 108-115.

18. Wagne, B., Roux, S., and Hild, F., Spectral approach to displacement evaluation from image analysis. European Physical Journal-Applied Physics, 2002. 17(3): p. 247-252.

19. Zienkiewicz, O.C. and Taylor, R.L., The finite element method. 5th ed. 2000, Oxford ; Boston: Butterworth-Heinemann. 
20. Rethore, J., Hild, F., and Roux, S., Shear-band capturing using a multiscale extended digital image correlation technique. Computer Methods in Applied Mechanics and Engineering, 2007. 196(49-52): p. 5016-5030.

21. Sutton, M.A., Orteu, J.-J., and Schreier, H.W., Image correlation for shape, motion and deformation measurements : basic concepts, theory and applications. 2009, New York, N.Y.: Springer. xx, $321 \mathrm{p}$.

22. Baker, S. and Matthews, I., Lucas-Kanade 20 years on: $A$ unifying framework. International Journal of Computer Vision, 2004. 56(3): p. 221-255.

23. Press, W.H., Numerical recipes : the art of scientific computing. 3rd ed. 2007, Cambridge, UK ; New York: Cambridge University Press. xxi, 1235 p.

24. Sacks, J., et al., Design and Analysis of Computer Experiments. Statistical Science,, 1989. 4(4): p. 409-435.

25. Matheron, G., Principles of geostatistics. Econimic Geology, 1963. 58: p. 1246-1266.

26. Forrester, A.I.J., Keane, A.J., and Bressloff, N.W., Design and analysis of "Noisy" computer experiments. Aiaa Journal, 2006. 44(10): p. 2331-2339.

27. Banerjee, K.S. and Carr, R.N., Ridge Regression - Biased Estimation for NonOrthogonal Problems. Technometrics, 1971. 13(4): p. 895-\&.

28. Tikhonov, A.N., Numerical methods for the solution of ill-posed problems. Mathematics and its applications. 1995, Dordrecht ; Boston: Kluwer Academic Publishers. ix, $253 \mathrm{p}$.

29. Forrester, A.I.J., Sóbester, A.S., and Keane, A.J., Engineering design via surrogate modelling : a practical guide. 2008, Chichester, West Sussex, England ; Hoboken, NJ: J. Wiley. xviii, $210 \mathrm{p}$.

30. Pan, B., Bias error reduction of digital image correlation using Gaussian pre-filtering. Optics and Lasers in Engineering, 2013. 51(10): p. 1161-1167.

31. Zhou, Y., et al., Image pre-filtering for measurement error reduction in digital image correlation. Optics and Lasers in Engineering, 2014. In Press.

32. Jones, D.R., A taxonomy of global optimization methods based on response surfaces. Journal of Global Optimization, 2001. 21(4): p. 345-383.

33. Schonlau, M., Computer Experiments and Global Optimization. 1997, University of Waterloo: Waterloo, Ontario, Canada.

34. Keane, A.J. and Nair, P.B., Computational Approaches for Aerospace Design: The Pursuit of Excellence. Computational Approaches for Aerospace Design: The Pursuit of Excellence, 2005: p. 1-582.

35. Vendroux, G. and Knauss, W.G., Submicron deformation field measurements: Part 2. Improved digital image correlation. Experimental Mechanics, 1998. 38(2): p. 86-92.

36. Lagarias, J.C., et al., Convergence properties of the Nelder-Mead simplex method in low dimensions. Siam Journal on Optimization, 1998. 9(1): p. 112-147.

37. Zhao, J.Q., et al., Initial guess by improved population-based intelligent algorithms for large inter-frame deformation measurement using digital image correlation. Optics and Lasers in Engineering, 2012. 50(3): p. 473-490.

38. Lophaven, S.N. and Nielsen, H.B., DACE A Matlab Kriging Toolbox. 2002.

39. Forrester, A.I.J., Sobester, A., and Keane, A.J., Multi-fidelity optimization via surrogate modelling. Proceedings of the Royal Society a-Mathematical Physical and Engineering Sciences, 2007. 463(2088): p. 3251-3269.

40. DIC Challenge 2D Dataset http://www.sem.org/dic-challenge/.

41. Schreier, H.W., Braasch, J.R., and Sutton, M.A., Systematic errors in digital image correlation caused by intensity interpolation. Optical Engineering, 2000. 39(11): p. 2915-2921.

42. Lehmann, T.M., Gonner, C., and Spitzer, K., Survey: Interpolation methods in medical image processing. leee Transactions on Medical Imaging, 1999. 18(11): p. 1049-1075. 
43. Wang, Y.Q., et al., Quantitative Error Assessment in Pattern Matching: Effects of Intensity Pattern Noise, Interpolation, Strain and Image Contrast on Motion Measurements. Strain, 2009. 45(2): p. 160-178.

44. Zhou, P. and Goodson, K.E., Subpixel displacement and deformation gradient measurement using digital image/speckle correlation (DISC). Optical Engineering, 2001. 40(8): p. 1613-1620.

45. Zhou, Y.H. and Chen, Y.Q., Propagation function for accurate initialization and efficiency enhancement of digital image correlation. Optics and Lasers in Engineering, 2012. 50(12): p. 1789-1797.

46. Labeas, G., Pasialis, V., Lin, X., Patterson, E.A., On the validation of solid mechanics models using optical measurements and Zernike decomposition. journal of Strain Analysis, submitted. 


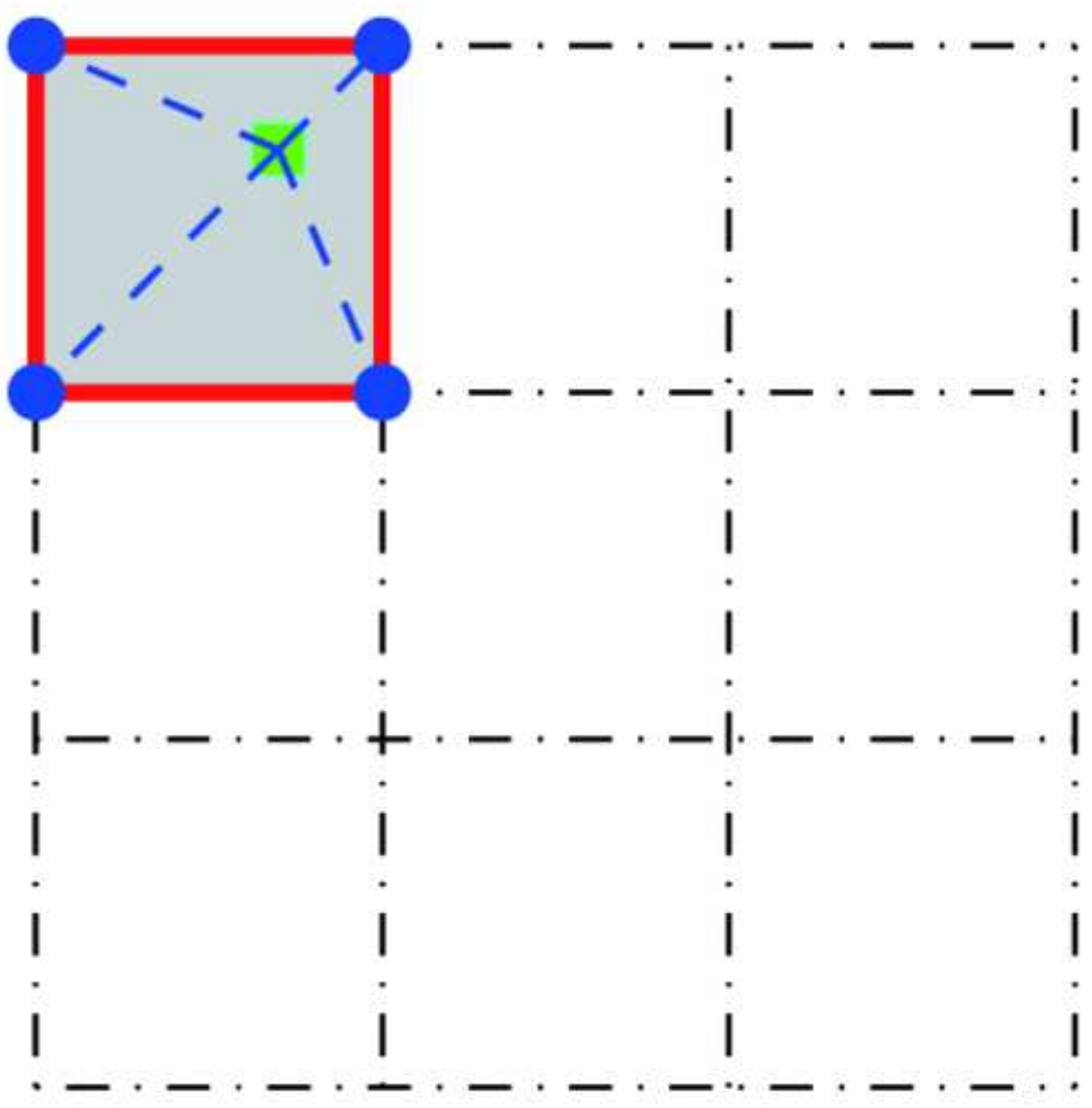




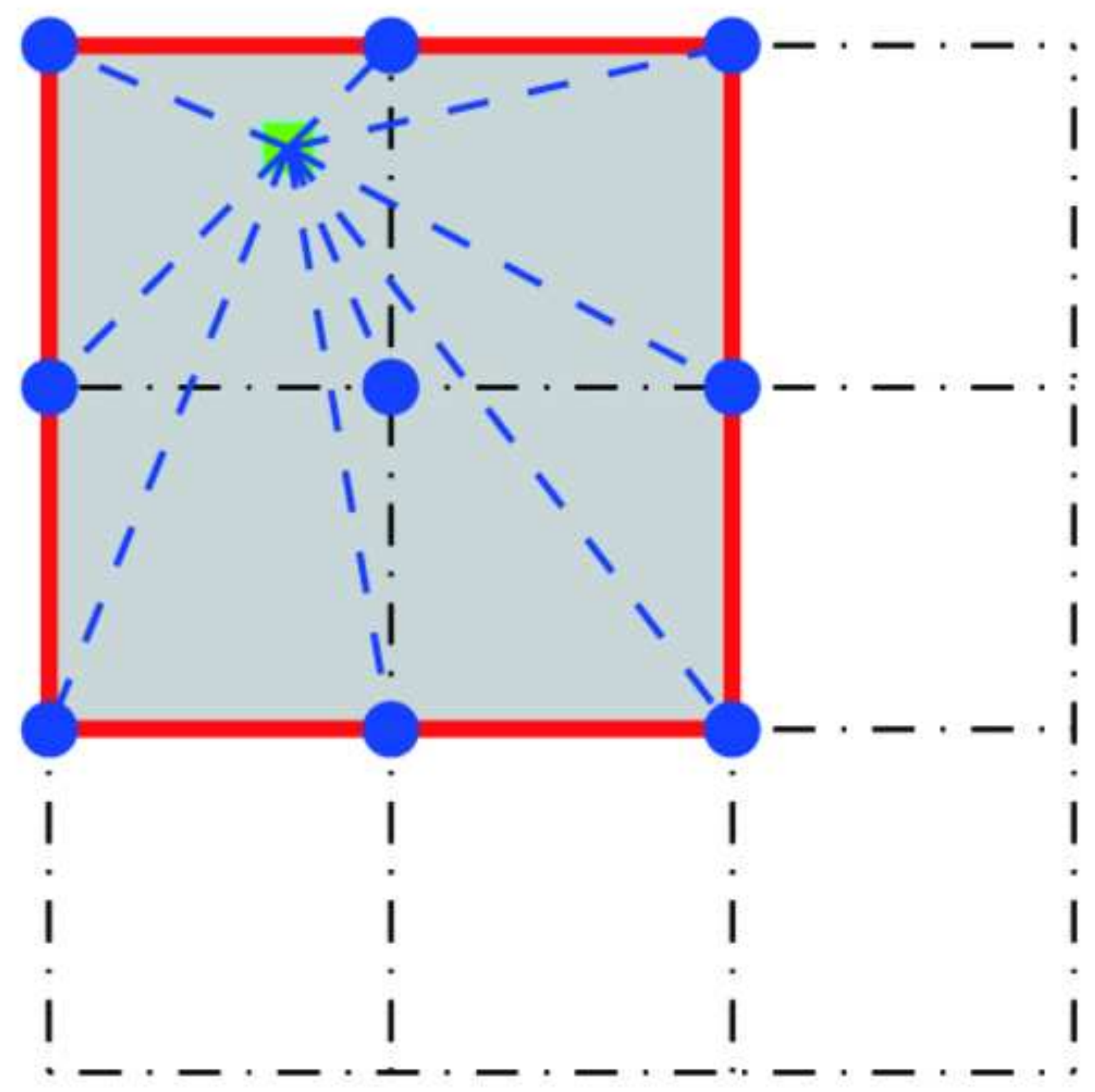


Click here to download high resolution image

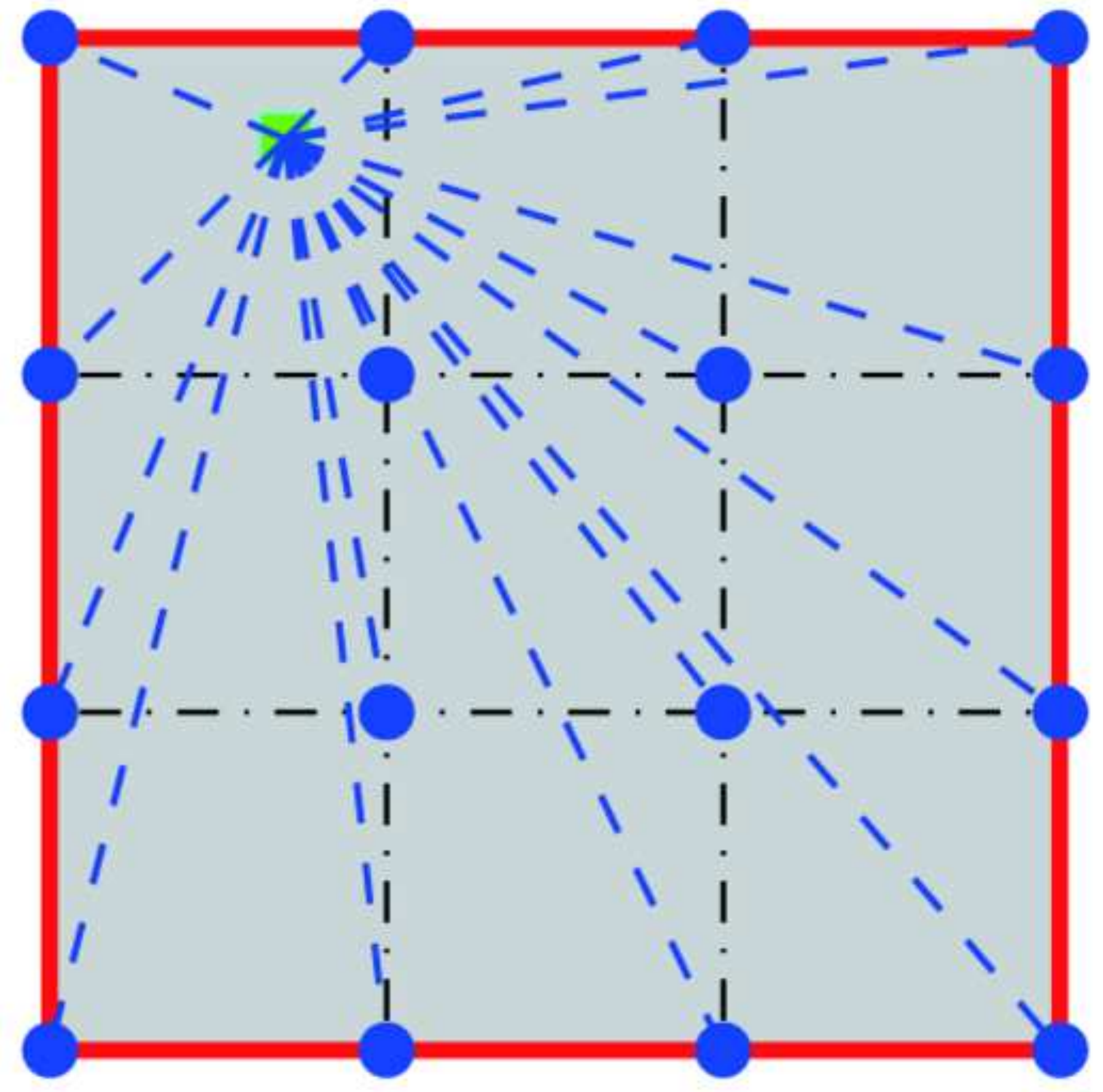


Click here to download high resolution image
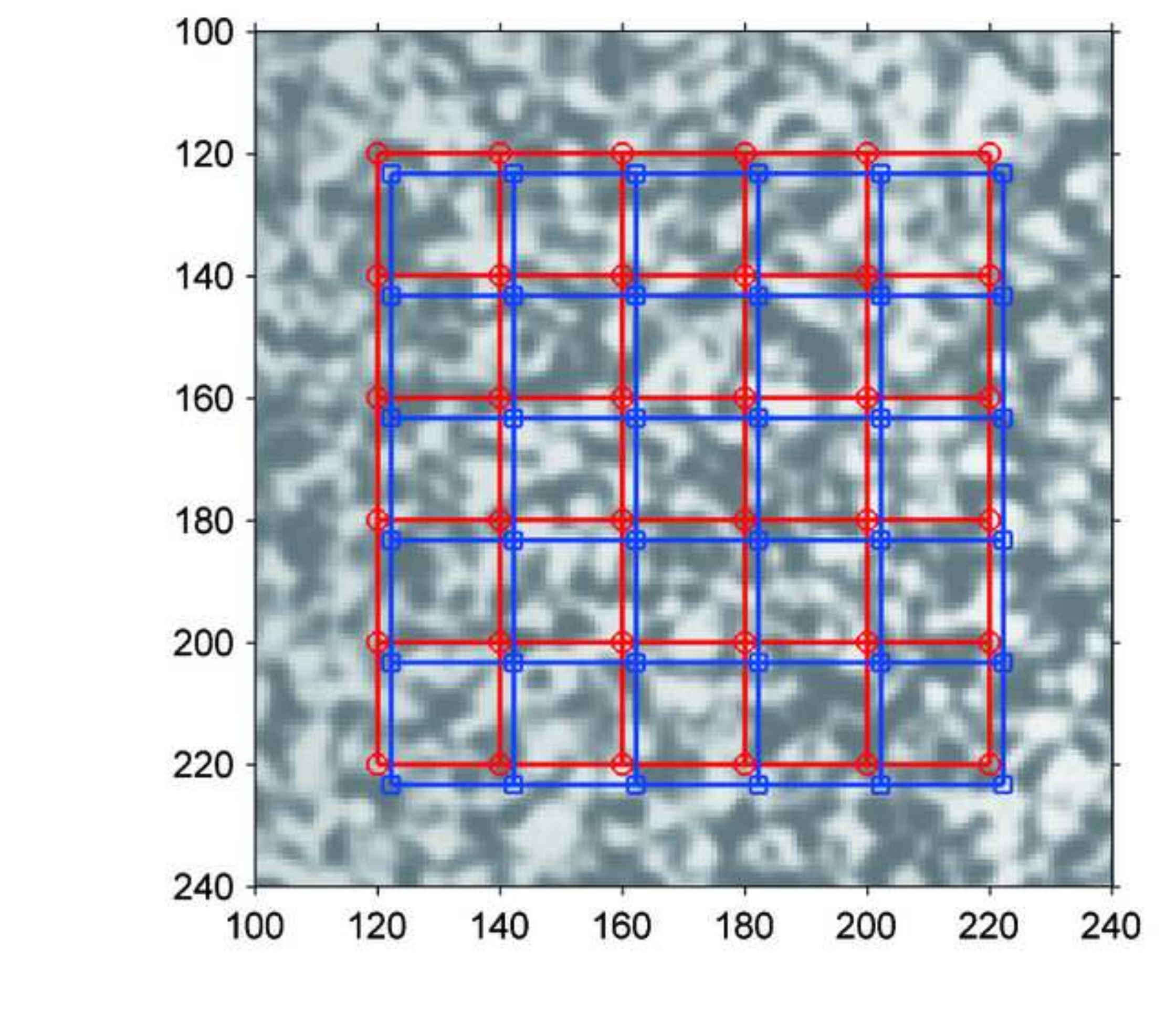


\section{Figure 3}

Click here to download high resolution image
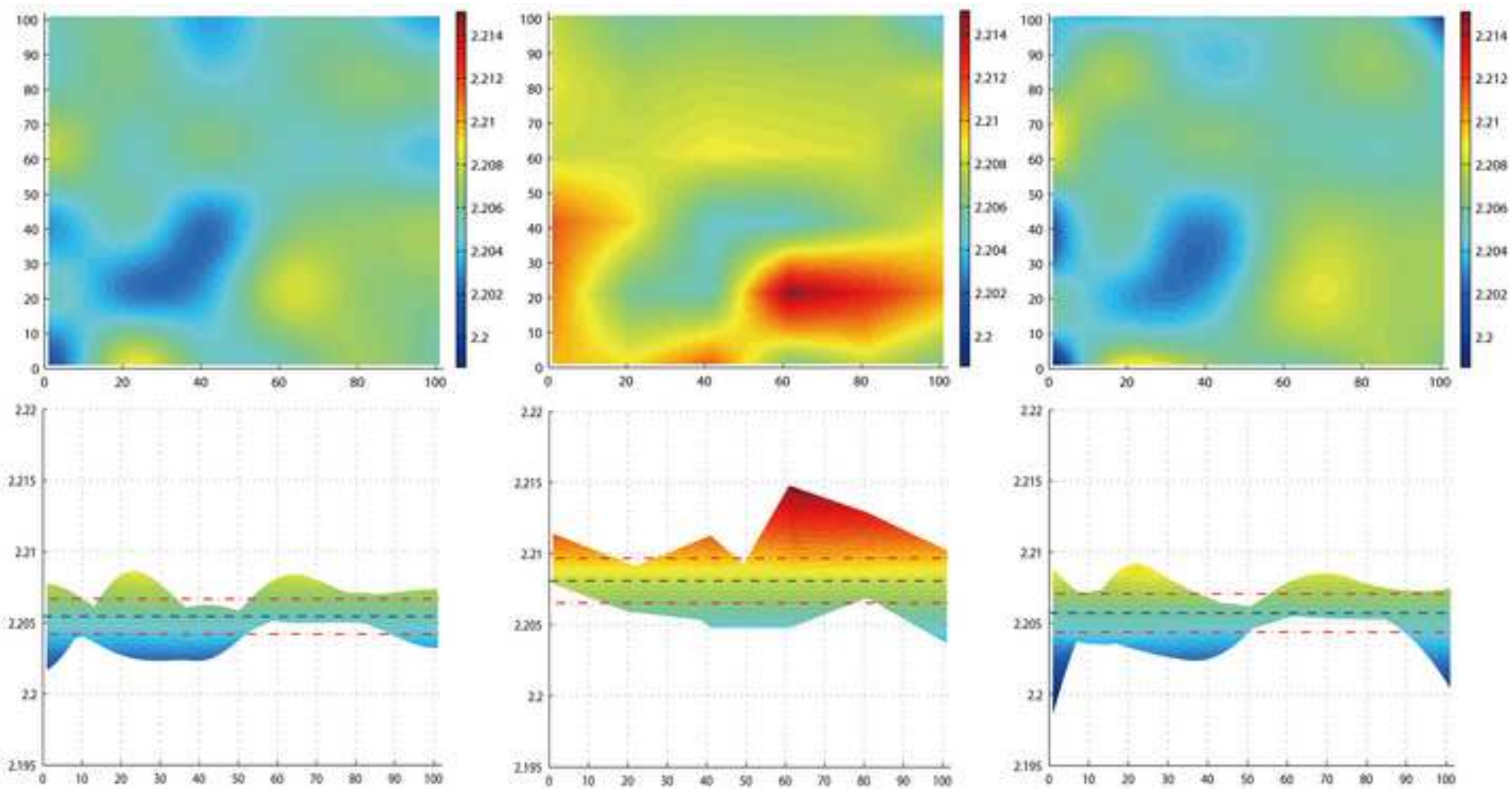


\section{Figure 4}

Click here to download high resolution image
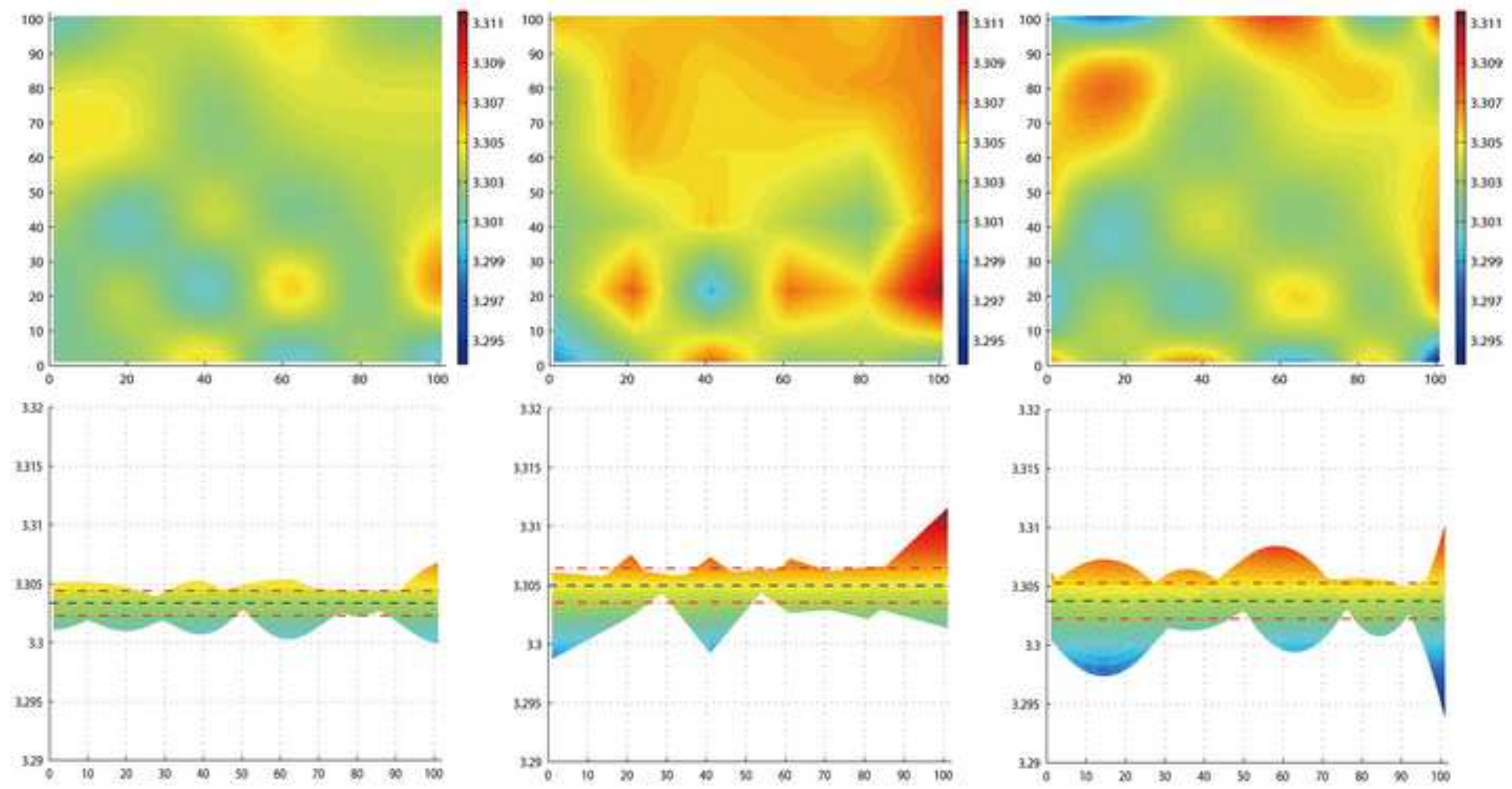

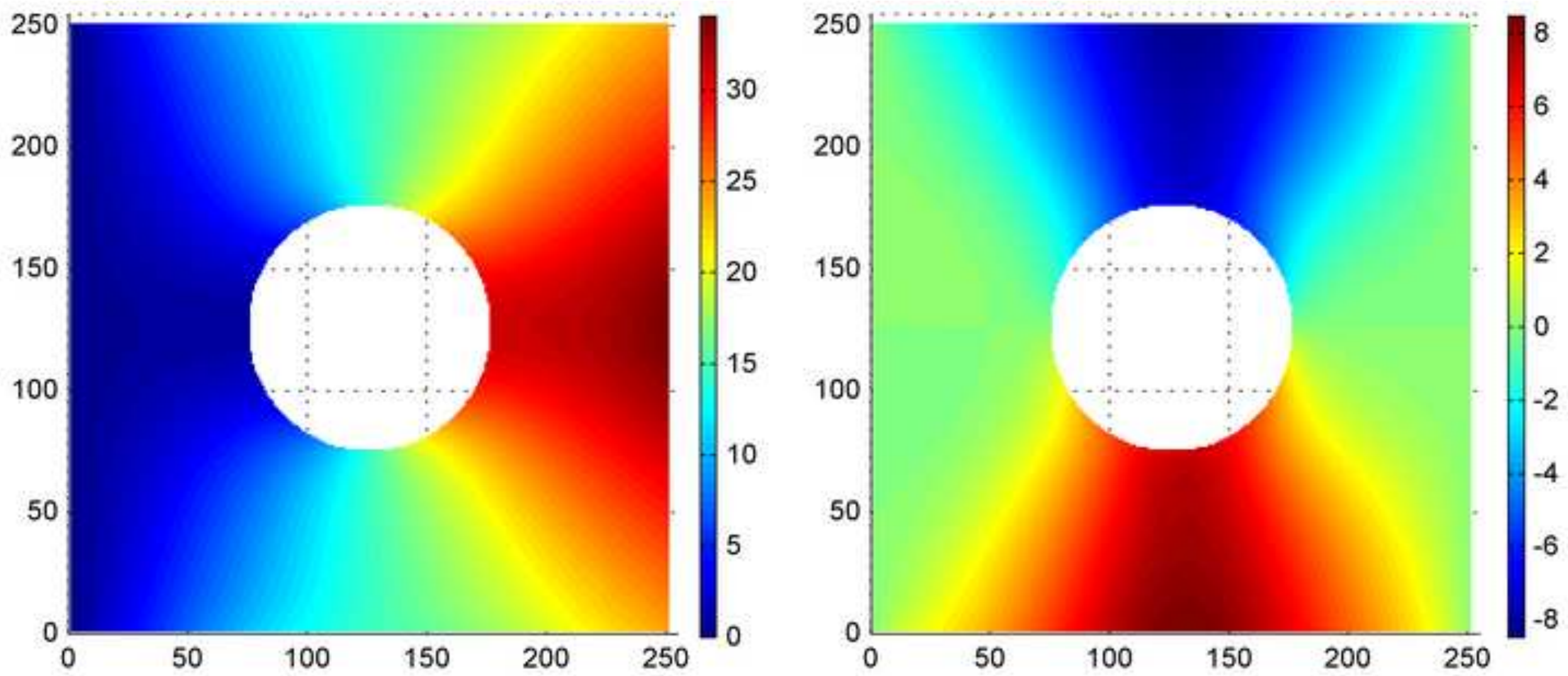

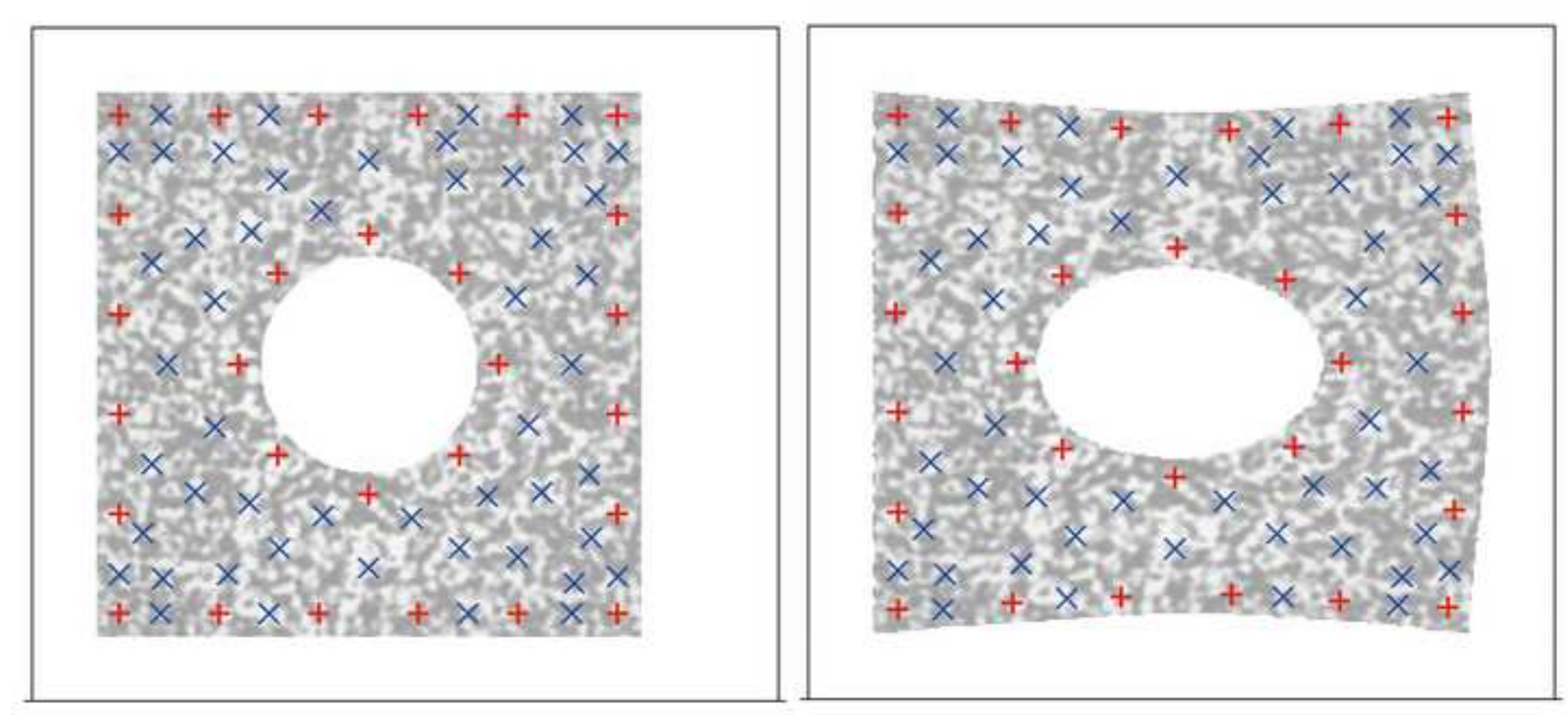
Figure 7

Click here to download high resolution image

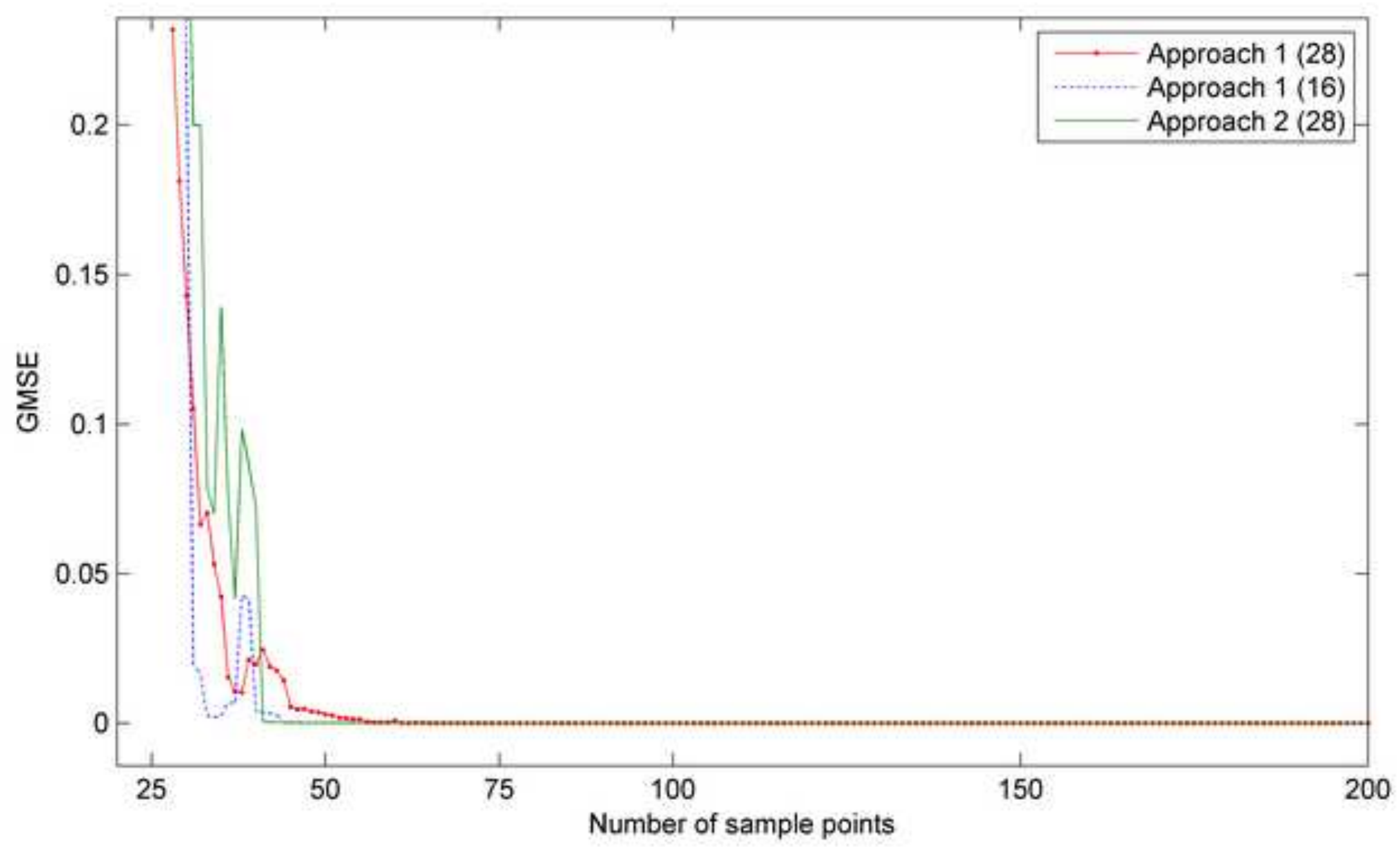




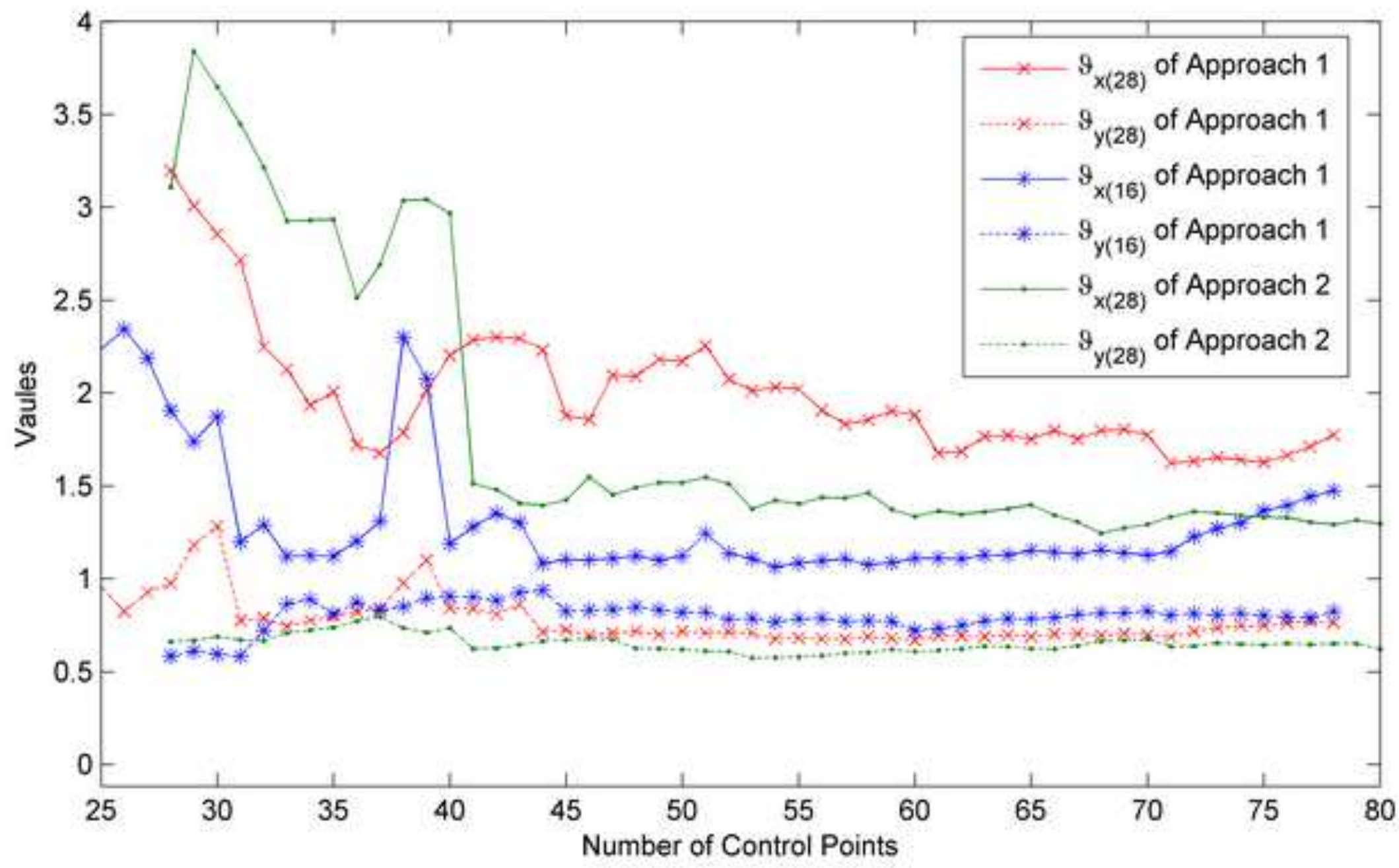


Click here to download high resolution image

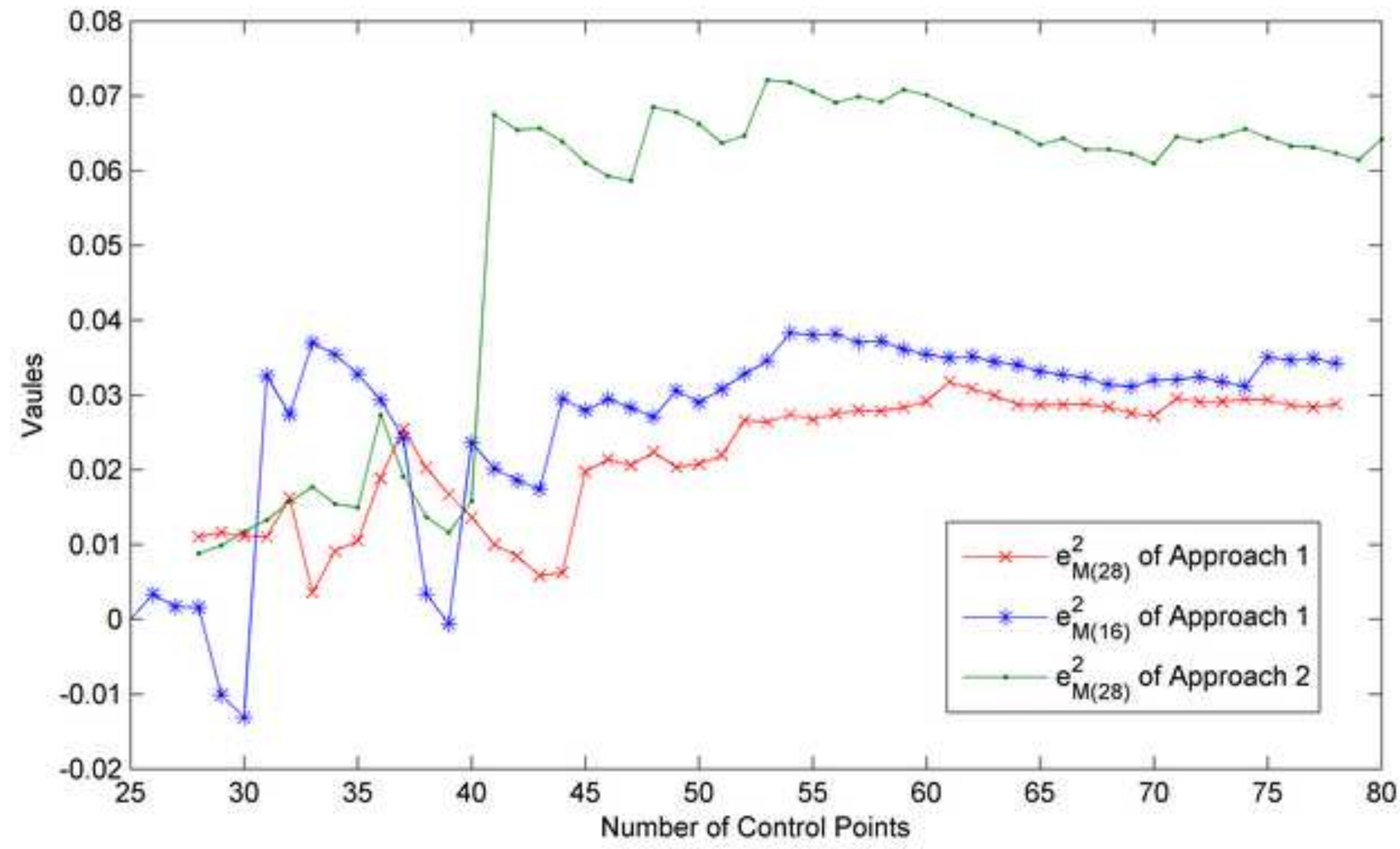


Click here to download high resolution image

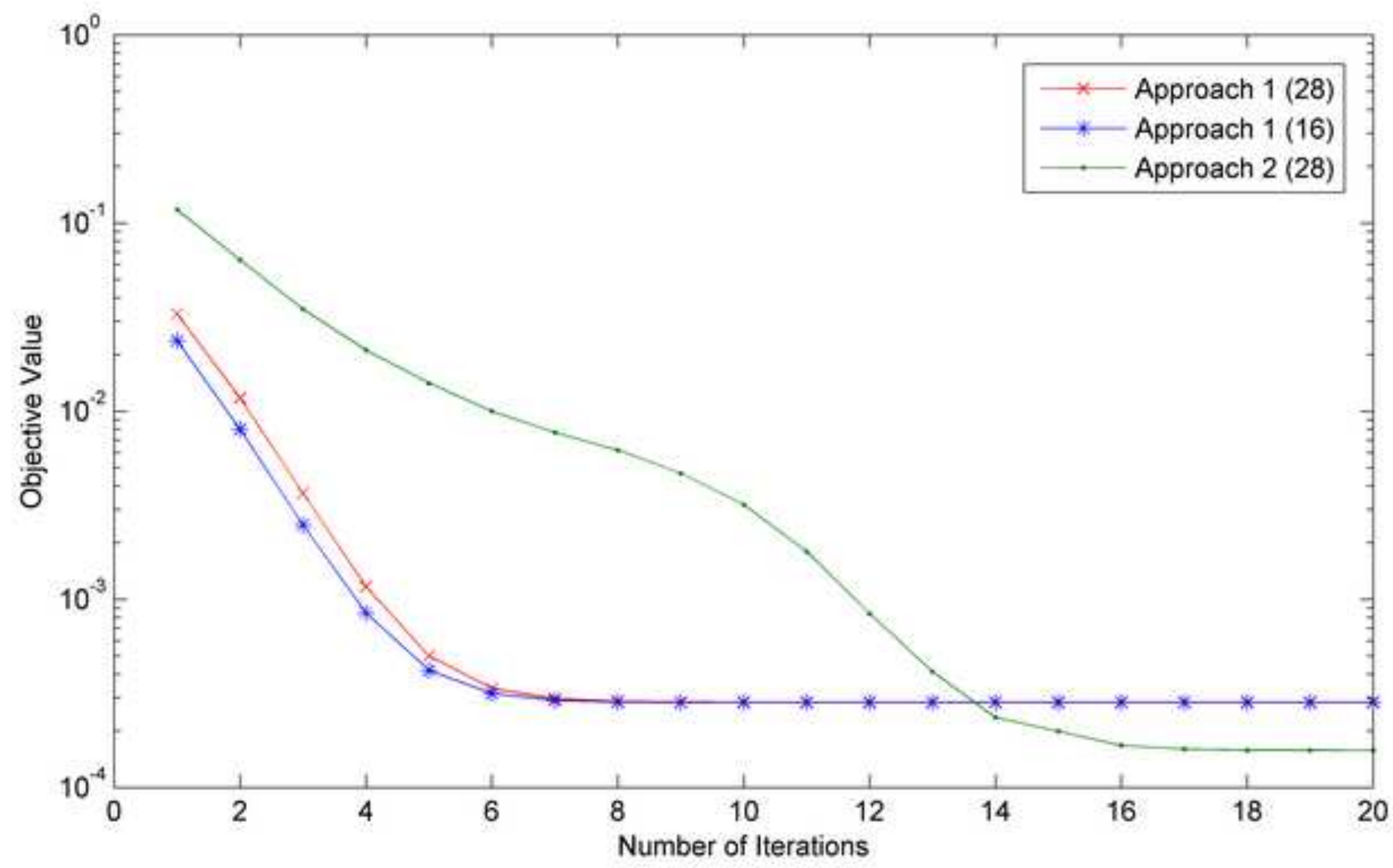


Click here to download high resolution image
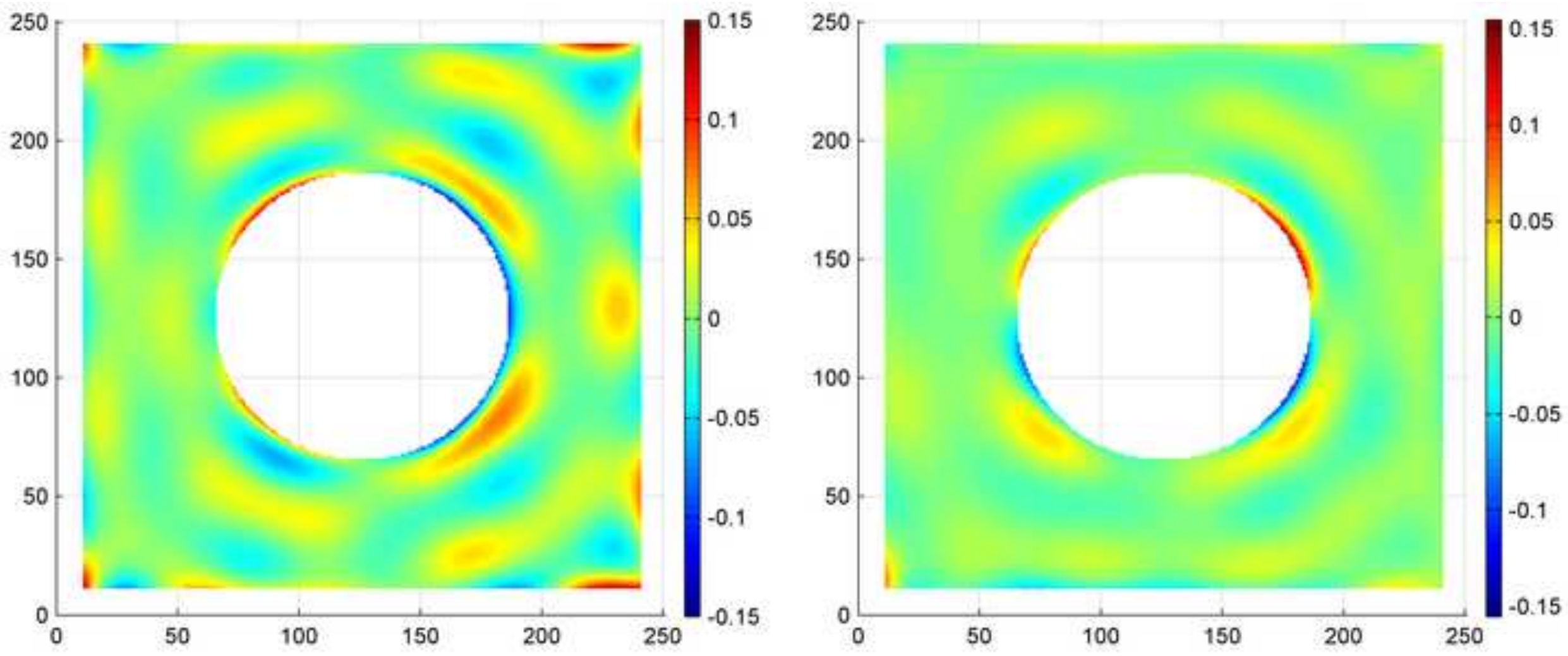
Click here to download high resolution image
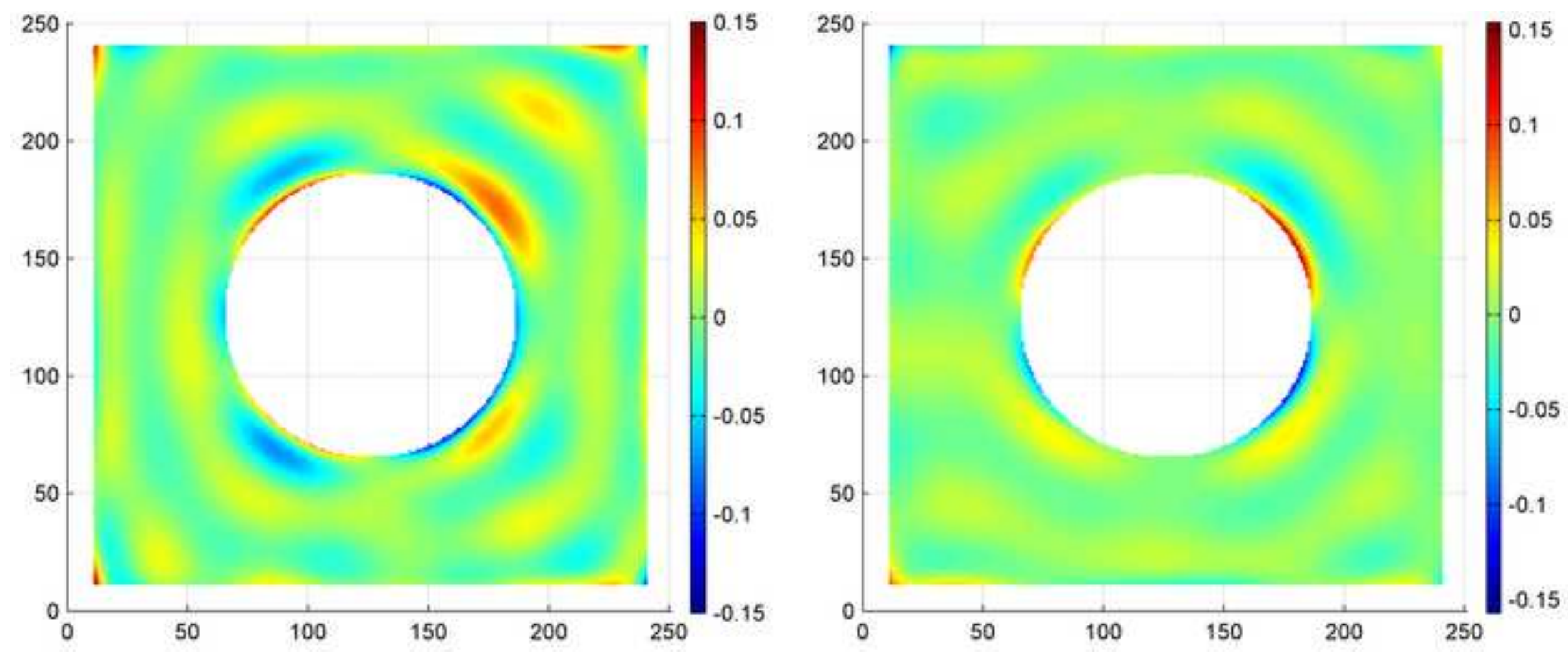
Click here to download high resolution image

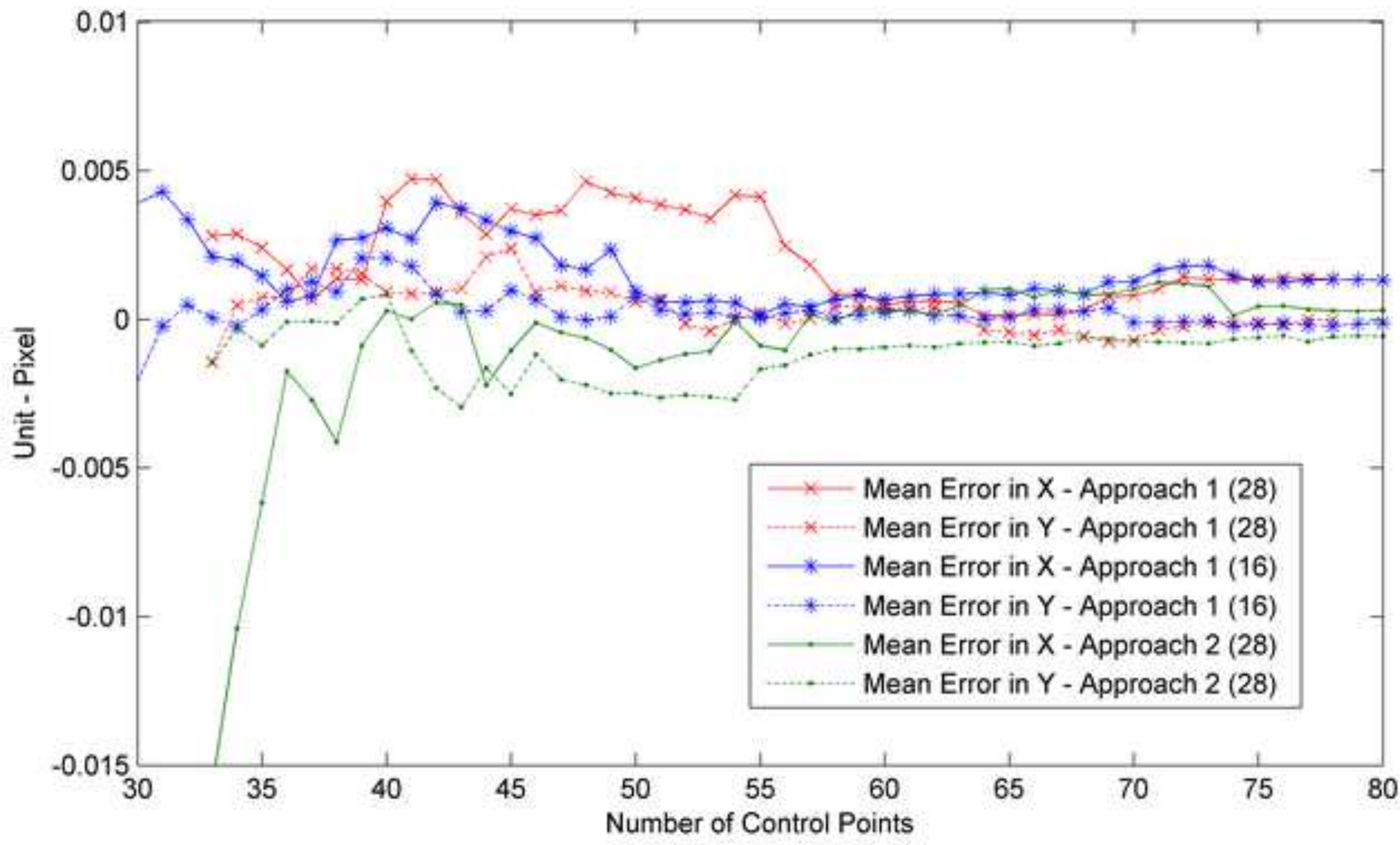




\section{Figure $12 b$}

Click here to download high resolution image

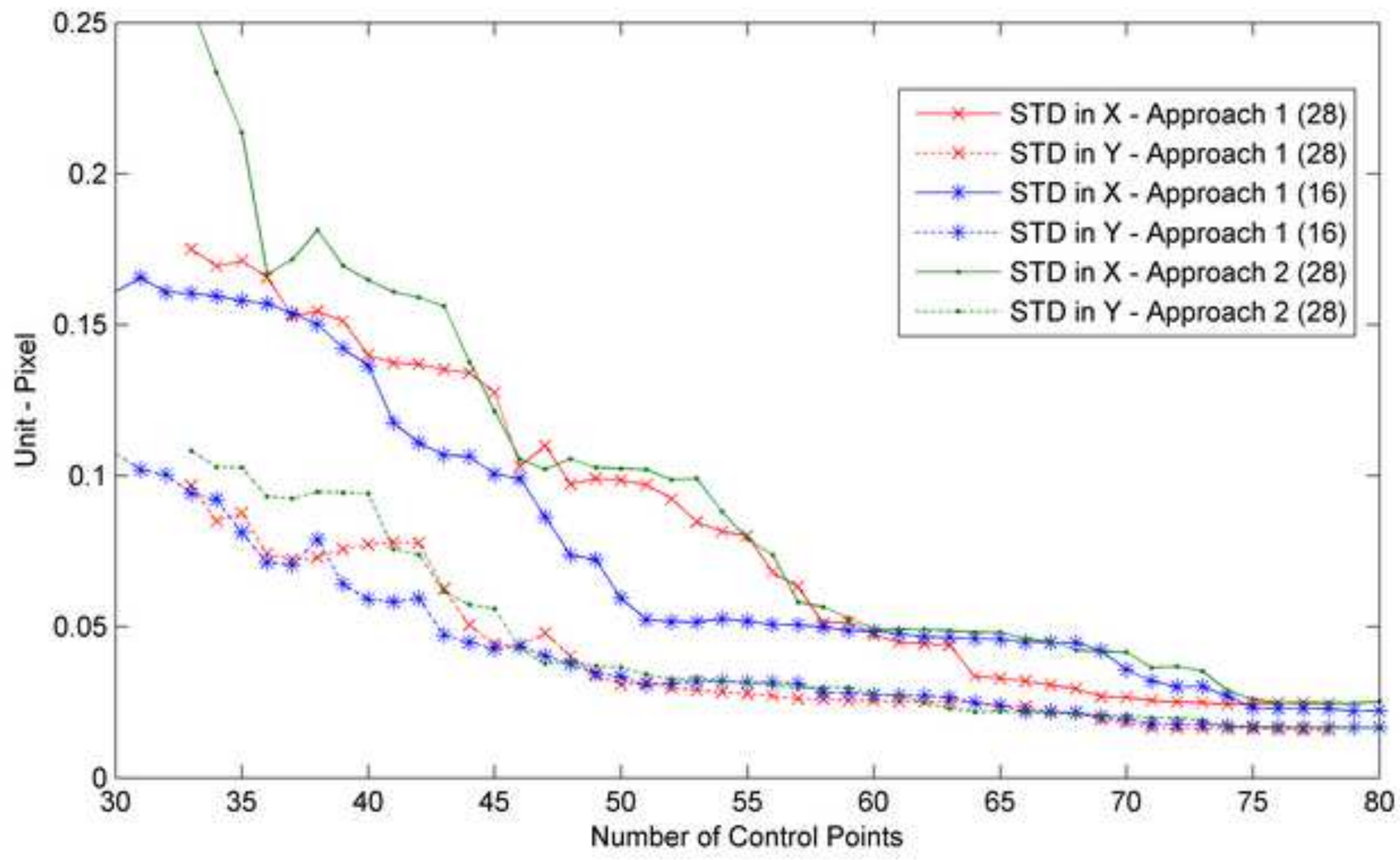


Click here to download high resolution image

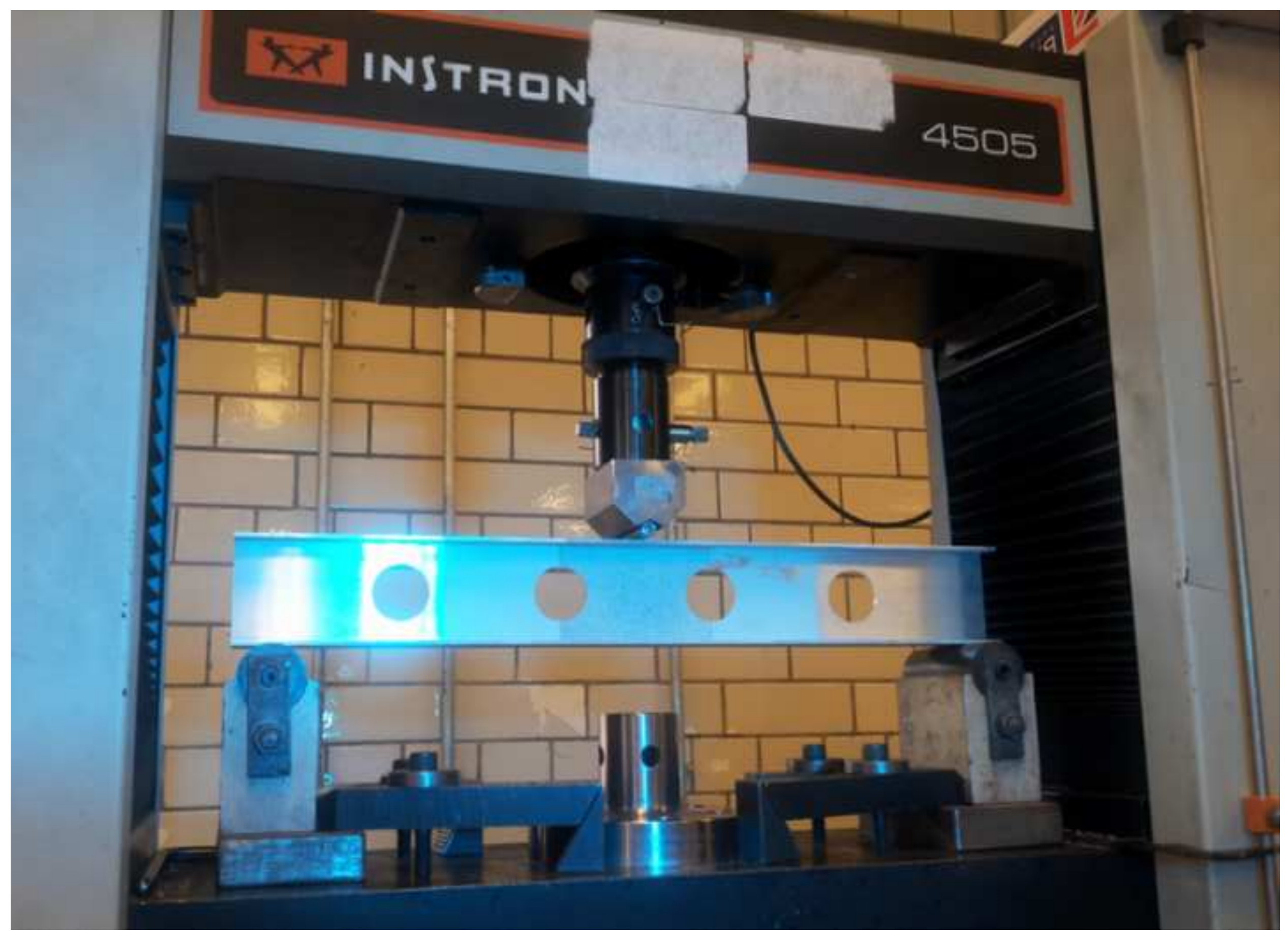




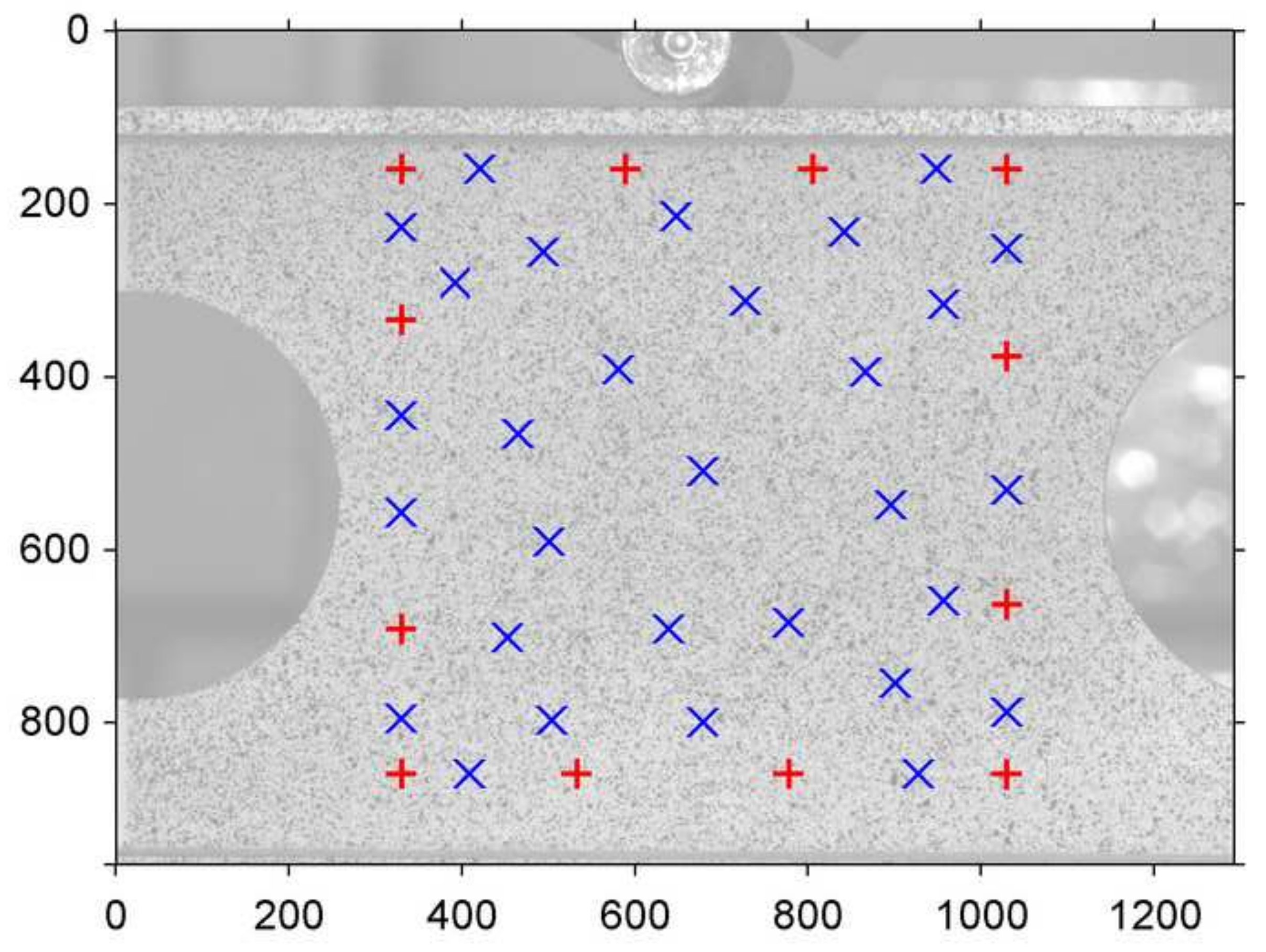


Click here to download high resolution image
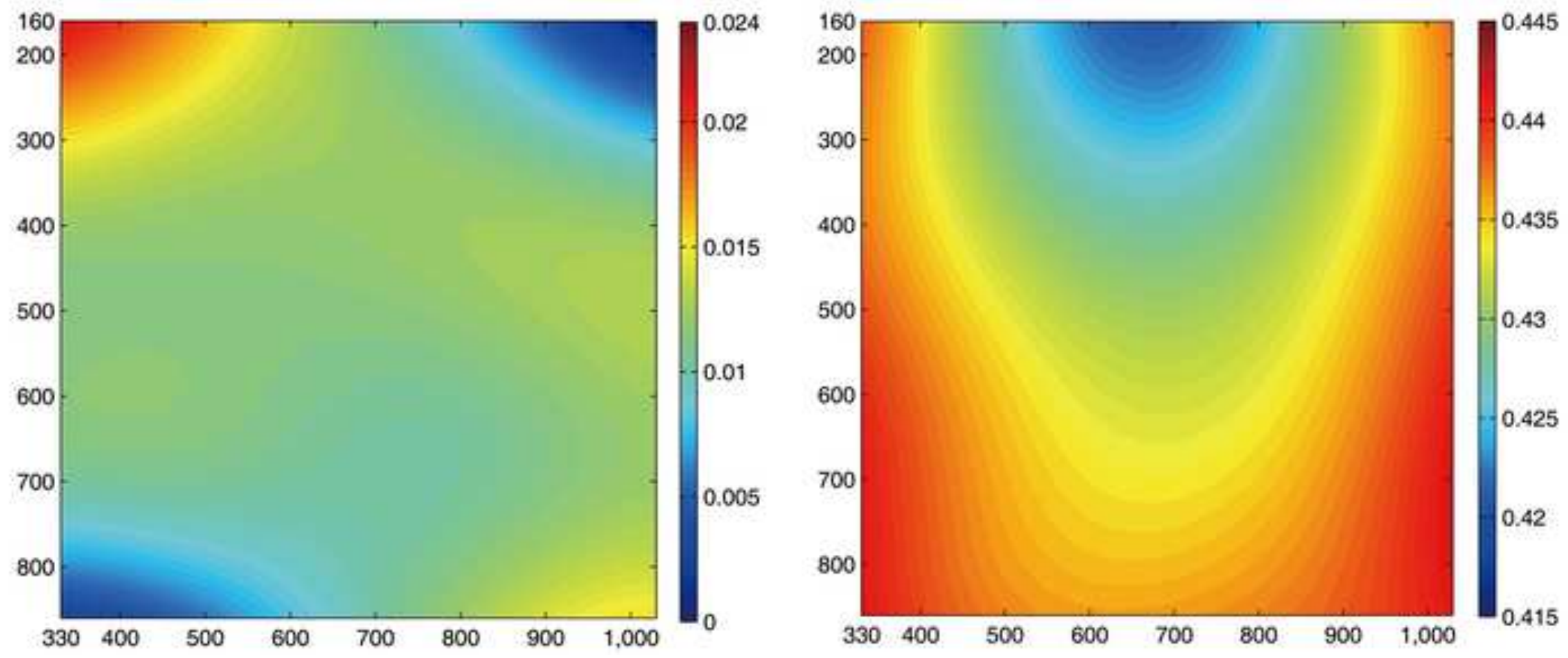
Click here to download high resolution image
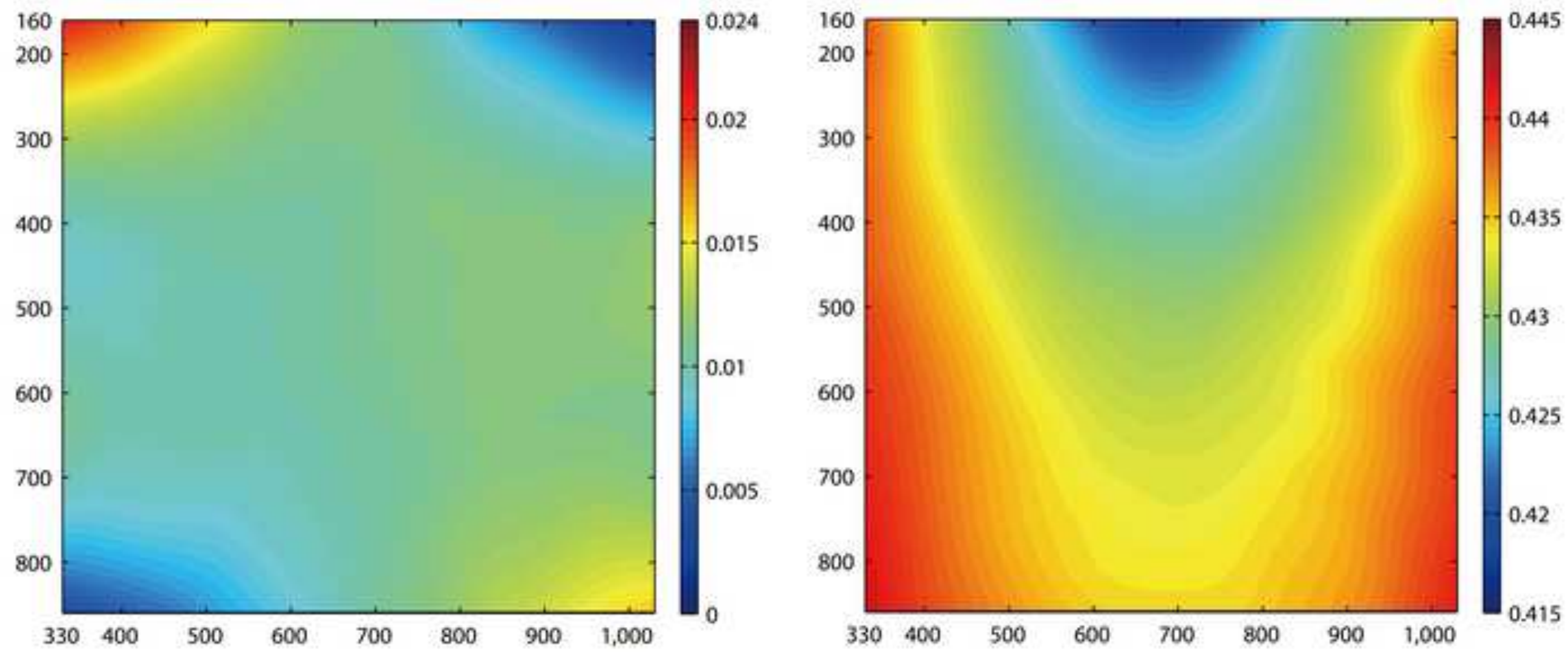
Click here to download high resolution image
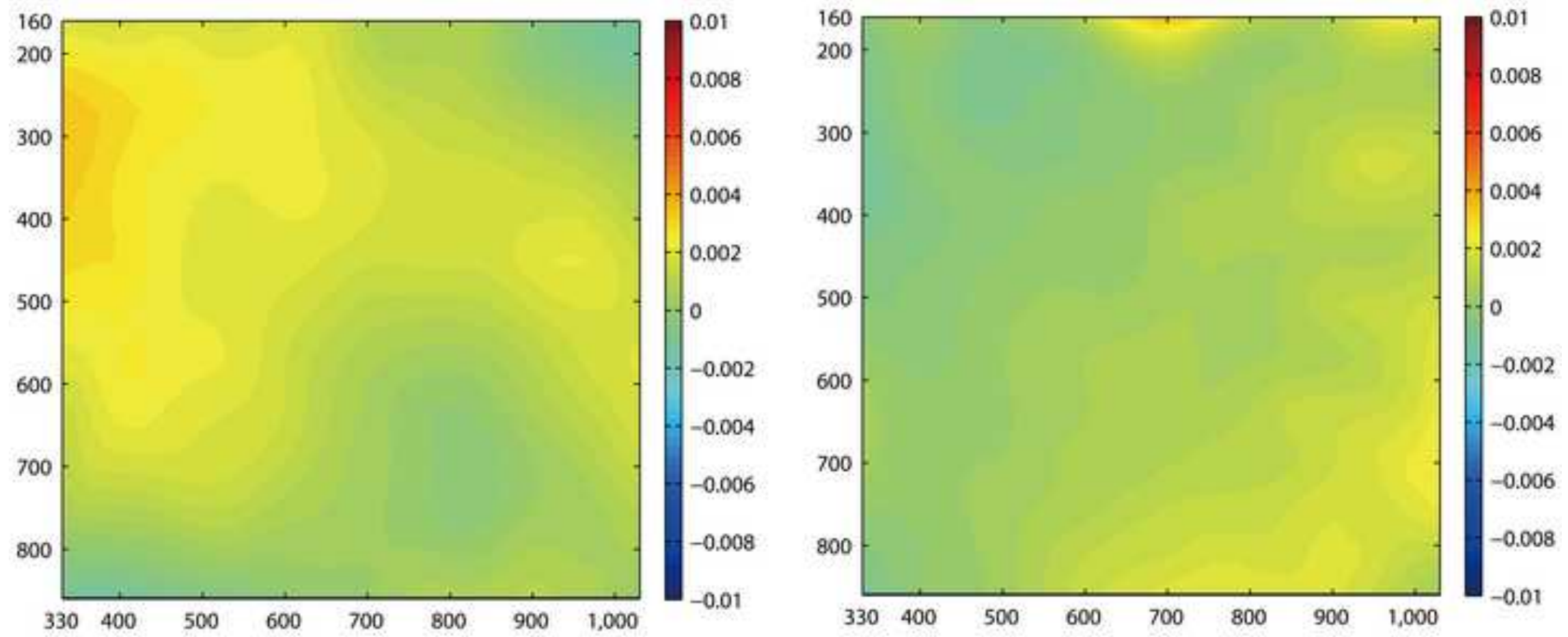
Table 1 Errors Comparison (unit pixel)

\begin{tabular}{|c|c|c|c|c|}
\hline \multicolumn{2}{|c|}{} & Kriging & Q4-FE & Cubic Spline \\
\hline \multirow{2}{*}{$\mathrm{X}$} & Mean Error & $5.44 \mathrm{e}-3$ & $8.10 \mathrm{e}-3$ & $5.72 \mathrm{e}-3$ \\
\cline { 2 - 5 } & STD & $1.23 \mathrm{e}-3$ & $1.57 \mathrm{e}-3$ & $1.33 \mathrm{e}-3$ \\
\hline \multirow{2}{*}{$\mathrm{Y}$} & Mean Error & $3.34 \mathrm{e}-3$ & $4.98 \mathrm{e}-3$ & $3.74 \mathrm{e}-3$ \\
\cline { 2 - 5 } & STD & $1.04 \mathrm{e}-3$ & $1.46 \mathrm{e}-3$ & $1.50 \mathrm{e}-3$ \\
\hline
\end{tabular}

* Mean Error here is the difference between actual Mean and the theoritical values i.e. $2.2 \& 3.3$ pixels 
Table 2 Measurement-error statistics in pixels.

\begin{tabular}{|c|c|c|c|c|}
\hline \multicolumn{2}{|c|}{} & $\begin{array}{c}\text { Approach 1 } \\
\text { (28 initial points \& 78 } \\
\text { in total) }\end{array}$ & $\begin{array}{c}\text { Approach 1 } \\
\text { (16 initial points \& 78 } \\
\text { in total) }\end{array}$ & $\begin{array}{c}\text { Approach 2 } \\
\text { (28 initial points \& 88 } \\
\text { in total) }\end{array}$ \\
\hline \multirow{3}{*}{ X } & $\begin{array}{c}\text { Mean } \\
\text { Error }\end{array}$ & 0.00135 & 0.00134 & $2.60 \mathrm{e}-4$ \\
\cline { 2 - 5 } & STD & 0.0244 & 0.0228 & 0.0209 \\
\hline \multirow{3}{*}{ Y } & $\begin{array}{c}\text { Mean } \\
\text { Error }\end{array}$ & $-1.51 \mathrm{e}-5$ & $-2.25 \mathrm{e}-4$ & $-4.93 \mathrm{e}-4$ \\
\cline { 2 - 5 } & STD & 0.0157 & 0.0166 & 0.0151 \\
\hline
\end{tabular}


Table 3 Mean values and standard deviations of the absolute difference

\begin{tabular}{|c|c|c|c|}
\hline \multicolumn{2}{|c|}{ Residual Disp } & Unit: $\mathrm{mm}$ & Unit: pixel* \\
\hline \multirow{2}{*}{$x$} & Mean & $1.3472 \mathrm{e}-3$ & 0.0182 \\
\cline { 2 - 4 } & STD & $9.1654 \mathrm{e}-4$ & 0.0124 \\
\hline \multirow{2}{*}{$y$} & Mean & $6.0095 \mathrm{e}-4$ & 0.0081 \\
\cline { 2 - 4 } & STD & $7.3189 \mathrm{e}-4$ & 0.0099 \\
\hline
\end{tabular}

*1 pixel length $\approx 0.074 \mathrm{~mm}$ 